\title{
Graft-versus-Host-Erkrankung der Haut
}

\section{Graft-versus-Host-Disease of the Skin}

U. Hillen

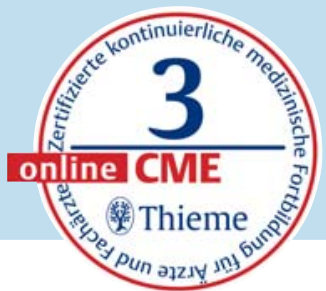

\section{Lernziele}

$\nabla$

Kenntnisse über:

- Klassifikation und Grading der Graftversus-Host-Erkrankung (GvHD)

- Organmanifestationen

- Klinik der kutanen GvHD

- Diagnostik der kutanen GvHD nach NIH-Konsensus

- Therapie der kutanen GvHD

\section{Einleitung}

\section{$\nabla$}

Hämatopoetische Stammzell-

\section{transplantation}

Innerhalb der letzten Jahrzehnte hat sich die hämatopoetische Stammzelltransplantation (SZT) zu einem wichtigen Therapieverfahren entwickelt. Unterschieden werden die autologe, die syngene und die allogene Stammzelltransplantation. Bei der autologen SZT werden dem Empfänger Stammzellen (SZ), meist im Rahmen einer vorausgehenden Chemotherapie, selbst entnommen, bei der syngenen SZT erfolgt die Übertragung von SZ von eineiigen Zwillingen. Bei der allogenen SZT werden die SZ von (verwandten oder nicht verwandten) Fremdspendern gewonnen. Die klassische Methode zur Gewinnung von hämatopoetischen Stammzellen ist die Entnahme von ca. $1000 \mathrm{ml}$ Knochenmarkblut durch multiple Beckenkammpunktionen beim Spender. Seit Mitte der 1990er-Jahre wird Knochenmark als Stammzellquelle jedoch zunehmend durch "mobilisierte“, d. h. in das Blut ausgeschwemmte SZ ersetzt (periphere Blutstammzellen). Hierzu wird dem Spender/Patienten G-CSF (granulozytenkoloniestimulierender Faktor) für 4-5 Tage subkutan injiziert. Vor der Übertragung der SZ erfolgt eine Hochdosischemotherapie und ggf. eine Ganzkörperbestrahlung. In der allogenen Transplantation nennt man diese Therapie „Konditionierung“. Die Konditionierung induziert eine Myeloablation und hat neben der antineoplastischen Wirkung die Aufgabe, durch Suppression des Empfänger- immunsystems eine Abstoßung des Transplantats zu verhindern [1]. Neben dieser klassischen Konditionierung werden auch weniger aggressive Konditionierungsschemata angewendet (sogenannte „Minitransplantation“), bei denen das primäre Ziel die Immunsuppression ist.

Die Anzahl der durchgeführten SZT ist in den vergangenen Jahren stetig gewachsen. Der Zuwachs ist im Wesentlichen bedingt durch die ansteigende Anzahl allogener SZT. So sind im 10-JahresZeitraum 1999-2009 die allogenen SZT von 1540 auf 2568 gestiegen (Ersttransplantationen 1416 bzw. 2376) [2]. Die autologe SZT zeigte im 10-Jahres-Zeitraum einen zeitweise schwankenden Verlauf, die autologen Ersttransplantationen lagen im Jahr 1999 bei 2221, im Jahr 2009 bei 2594. Die wesentlichen Indikationen für die allogene SZT sind Leukämien und maligne Lymphome ( $\bullet$ Tab. 1). Zugenommen haben die allogenen SZT bei akuten Leukämien, während die Indikation zur SZT bei chronischer myeloischer Leukämie durch die Therapiemöglichkeiten mit Tyrosinkinaseinhibitoren insgesamt stark abgenommen hat $[2,3]$. In den letzten 10 Jahren ist eine kontinuierliche Zunahme des Anteils der Transplantationen von unverwandten Spendern und die zunehmende Nutzung von Blutstammzellen im Vergleich zum Knochenmark als Stammzellquelle zu verzeichnen [2].

Da bei der allogenen SZT Gewebe verschiedener Individuen miteinander in dauerhaften Kontakt gebracht werden, entsteht ein Chimärismus. Jeder Mensch besitzt ein individuelles Muster an Oberflächenmolekülen, das primär für die Abstoßung unterschiedlicher Gewebe verantwortlich ist, die MHC-Moleküle (MHC: Major Histocompatibility Complex, Haupthistokompatibilitätskomplex). Der MHC wird auf dem kurzen Arm des Chromosoms 6 codiert. Man unterscheidet MHCKlasse-I-Moleküle (HLA-A, -B und -C), die auf praktisch allen kernhaltigen Zellen exprimiert werden, und die MHC-Klasse-II-Moleküle (HLADR, -DQ -DP), die nur auf antigenpräsentierenden Zellen vorhanden sind. Nach Möglichkeit wird die SZT mit HLA-identischen Spenderzellen
VNR

2760518011060000432

Bibliografie

Dol http://dx.doi.org/ 10.1055/s-0030-1256728 Akt Dermatol 2011; 37:

317-332 @ Georg Thieme Verlag KG Stuttgart · New York ISSN 0340-2541

Korrespondenzadresse PD Dr. Uwe Hillen

Klinik für Dermatologie Universitätsklinikum Essen Hufelandstr. 55

45147 Essen

uwe.hillen@uk-essen.de 
Tab. 1 Indikationen für die hämatopoetische allogene Stammzelltransplantation.

\begin{tabular}{|l|l|}
\hline Erkrankung & $\begin{array}{l}\text { Anteil an den in Deutschland } \\
\text { durchgeführten SZT }\end{array}$ \\
\hline akute myeloische Leukämie & $33,1 \%$ \\
\hline akute lymphatische Leukämie & $13,7 \%$ \\
\hline chronische myeloische Leukämie & $2,8 \%$ \\
\hline chronische lymphatische Leukämie & $4,2 \%$ \\
\hline myelodysplastisches Syndrom & $13,7 \%$ \\
\hline myeloproliferatives Syndrom & $5,4 \%$ \\
\hline Plasmazellerkrankungen (multiples Myelom u. a.) & $7,5 \%$ \\
\hline Morbus Hodgkin & $1,1 \%$ \\
\hline Non-Hodgkin-Lymphome & $10,6 \%$ \\
\hline solide Tumore & $0,9 \%$ \\
\hline Knochenmarksaplasie/aplastische Anämie & $2,7 \%$ \\
\hline Hämoglobinopathien & $0,8 \%$ \\
\hline angeborene Metabolismusdefekte & $2,4 \%$ \\
\hline primäre Immundefekte & $0,7 \%$ \\
\hline *Angaben des Deutsches Registers für Stammzelltransplantation für das Jahr 2009 [2]
\end{tabular}

vorgenommen. Unter Geschwistern besteht eine Wahrscheinlichkeit von 25\%, einen genotypisch geeigneten HLA-identischen Spender zu finden. Falls keine HLA-identischen Geschwister zur Verfügung stehen, muss nach einem phänotypisch identischen bzw. partiell HLA-kompatiblen Familienspender oder HLA-kompatiblen Fremdspender aus nationalen und internationalen Knochenmarkspenderegistern gesucht werden.

Die Anzahl der hämatopoetischen Stammzelltransplantationen ist in den vergangenen Jahren stetig gewachsen. Der Anteil der Transplantationen von unverwandten Spendern und die Nutzung von Blutstammzellen nehmen zu.

\section{Graft-versus-Host-Erkrankung Pathophysiologie}

Die Voraussetzungen dafür, dass eine GvHD entstehen kann, wurden 1966 von Billingham definiert [4]:

1. Das Transplantat muss immunkompetente Zellen enthalten.

2. Der Empfänger weist Gewebeantigene auf, die der Transplantatspender nicht exprimiert.

3. Der Empfänger ist nicht in der Lage, die Spenderzellen zu inaktivieren bzw. zu eliminieren.

Damit eine GvHD entsteht, muss das Transplantat immunkompetente Zellen enthalten, der Empfänger Gewebeantigene aufweisen, die der Transplantatspender nicht exprimiert, und der Empfänger nicht in der Lage sein, die Spenderzellen zu inaktivieren bzw. zu eliminieren.

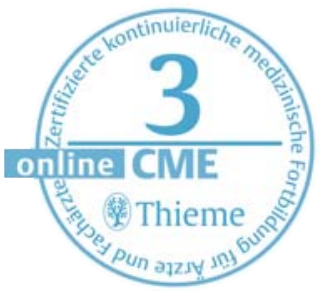

Die immunkompetenten Zellen, die maßgeblich für die Entwicklung der GvHD sind, sind T-Zellen. Die Gewebeantigene, die die Donorzellen nicht exprimieren, sind disparate HLA-Moleküle. Das Ziel der allogenen SZT ist, die Spenderzellen im Empfängerorganismus zu etablieren. Dies ist nicht nur wichtig für die Rekonstitution der Immunabwehr, sondern auch zur Aufrechterhaltung der Rezidivfreiheit. T-Zellen und NK-Zellen (natürliche Killerzellen) bewirken den sogenannten GvL-Effekt (GvL: Graft versus Leukemia). Der genaue Mechanismus des GvL-Effekts ist bisher nicht vollständig bekannt, doch agieren die Spenderzellen naturgemäß auch gegen die Tumorzellen, die fremde Gewebeantigene tragen, und können diese eliminieren [5]. Somit werden bei einer allogenen SZT bewusst die Voraussetzungen für die Entwicklung einer GvHD geschaffen, und ein gewisses Maß an GvHD wird unter dem Aspekt des gleichzeitigen GvL-Effekts in Kauf genommen. Auch bei HLA-identischer SZT entwickelt sich bei etwa $40 \%$ der Patienten eine akute GvHD (aGvHD), die eine hoch dosierte Kortikosteroid-Therapie erfordert [6]. Verantwortlich dafür sind die Minor-Histokompatibilitätsantigene (MiHA). Dabei handelt es sich um von polymorphen Genen codierte Proteine, die den Empfänger vom Donor unterscheiden. Ein Beispiel dafür sind auf dem Y-Chromosom liegende Gene, die sich von dem Homolog auf dem X-Chromosom stark unterscheiden und spezifisch männliche H-Y-Antigene bilden [7]. Die MiHAs H-Y und HA-3 werden auf allen Geweben exprimiert und stellen daher ein Ziel sowohl in der Entwicklung der GvHD als auch des GvL-Effekts dar [6]. MiHAs können auch durch Gene entstehen, die vom Empfänger, aber nicht vom Donor exprimiert werden oder durch eine unterschiedliche posttranslationale Modifikation. Die Expression von MiHAs kann gewebeabhängig sein. So werden HA-1 und HA-2 überwiegend auf Zellen des hämatopoetischen Systems exprimiert und sind daher eine Zielstruktur für den GvL-Effekt $[6,7]$.

Die Erkennung von Gewebeantigenen des Empfängers durch Spender-T-Zellen ist eine Voraussetzung für den Anti-Tumoreffekt.

\section{Klassifikation}

Die GvHD wird in eine akute und eine chronische GvHD (cGvHD) unterteilt. Bis vor Kurzem wurde die Grenze zwischen aGvHD und cGvHD bei Tag 100 gesetzt. Im Jahr 2005 gab eine Arbeitsgruppe des National Institute of Health (NIH) eine Konsensusempfehlung heraus, wonach die Differenzierung zwischen aGvHD und cGvHD auf Basis von klinischen Kriterien und dem zeitlichen Abstand zwischen Auftreten der Symptome und der SZT bzw. Donor-Lymphozyten-Infusion (DLI) erfolgt [20]. Die klassische aGvHD wird definiert als Erkrankung, die bis Tag 100 post transplantationem auftritt und ausschließlich klinische Zeichen der aGvHD zeigt. Die persistierende, rezidivierende oder verzögert auftretende aGvHD entwickelt sich nach Tag 100 mit ebenfalls ausschließlich klinischen Zeichen der aGvHD. Für die cGvHD wird kein Zeitlimit gesetzt, sie kann sich als klassische cGvHD manifestieren, wenn ausschließlich klinische Zeichen der cGvHD vor- 
liegen. Ein Erkrankungsbild, das sowohl Zeichen der aGvHD als auch der CGvHD aufweist, wird als Overlap-Syndrom bezeichnet ( $\bullet$ Tab. 2).

Die GvHD wird eingeteilt in eine klassische aGvHD, in eine persistierende, rezidivierende oder verzögert auftretende aGvHD, in eine klassische cGvHD und in ein Overlap-Syndrom.

\section{Akute GvHD}

In der Pathophysiologie der aGvHD werden 3 Phasen unterschieden [6]:

1. Aktivierung antigenpräsentierender Zellen des Empfängers

2. Aktivierung von Donor-T-Zellen

3. Effektorphase

Die Konditionierung führt zu einer Gewebeschädigung mit Freisetzung proinflammatorischer Zytokine, wie Tumor-Nekrose-Faktor alpha (TNF$\alpha$ ), IL-1 oder IL-6 (IL: Interleukin). Das führt zu einer Aktivierung der antigenpräsentierenden Zellen des Empfängers mit Hochregulation der HLA-Moleküle, kostimulatorischer Moleküle und Adhäsionsmoleküle. Durch die Translokation von Bakterien durch die geschädigte Barriere des Gastrointestinaltrakts werden endotoxisch wirksame Lipopolysaccharide (LPS) eingeschwemmt, die ebenfalls eine Aktivierung der antigenpräsentierenden Zellen bewirken. Frühere Arbeiten konnten zeigen, dass eine ineffektive Darmdekontamination anaerober Bakterien ein Risikofaktor für das Auftreten einer aGvHD darstellt [8].

In den 1990er-Jahren wurden Toll-like-Rezeptoren entdeckt, die zu der Gruppe der PRR (pattern recognition receptors) gehören und PAMP (pathogen associated molecular patterns) auf Erregern erkennen. Die Aktivierung von PRR führt u. a. zu einer Aktivierung von dendritischen Zellen (Phagozytose, Zykokinsekretion, Migration in drainierende Lymphknoten). Neben denTLR wurden weitere PRR entdeckt, wie intrazelluläre NOD-likeRezeptoren (NLR). NLR sind ebenfalls an der Sekretionsregulation von proinflammatorischen Zytokinen, wie IL-1 beta und IL-18, beteiligt. TLR und NLR haben auch eine Bedeutung für die Entwicklung der GvHD. Es konnte gezeigt werden, dass Polymorphismen der für NOD-2 und TLR-4 codierenden Gene mit einer höheren Inzidenz einer GvHD assoziiert waren [9].

In der 2. Phase werden innerhalb des entstandenen inflammatorischen Milieus Donor-T-Zellen aktiviert. Dies führt zu einer Sekretion von T-Helferzellen-1-Zytokinen (IL-2, Interferon gamma [IFN- $\gamma]$, TNF- $\alpha$ ) und einer weiteren Aktivierung von T-Zellen und natürlichen Killerzellen. Das bedeutsamste Zytokin ist dabei IL-2. Die Wirkung von IFN- $\gamma$ ist pleiotop. Einerseits fördert IFN- $\gamma$ die GvHD via einer verstärkten Expression von MHC-Molekülen, Chemokinrezeptoren und Adäsionsmolekülen sowie einer erhöhten Sensitivität von Makrophagen, z.B. gegenüber LPS, anderer-
Tab. 2 Klassifizierung der GvHD nach NIH-Konsensus [nach 20].

\begin{tabular}{|llll|}
\hline Kategorie & $\begin{array}{l}\text { Beginn der } \\
\text { Symptome nach } \\
\text { SZT oder DLI }\end{array}$ & $\begin{array}{l}\text { Zeichen der } \\
\text { akuten GvHD }\end{array}$ & $\begin{array}{l}\text { Zeichen der } \\
\text { chronischen } \\
\text { GvHD }\end{array}$ \\
\hline $\begin{array}{l}\text { akute GvHD } \\
\text { klassische akute GvHD }\end{array}$ & 100 Tage & ja & nein \\
\hline $\begin{array}{l}\text { persistierende, } \\
\text { rezidivierende oder } \\
\text { späte akute GvHD } \\
\text { chronische GvHD }\end{array}$ & ja & nein \\
\hline $\begin{array}{l}\text { klassische chronische } \\
\text { GVHD }\end{array}$ & kein Zeitlimit & nein & ja \\
\hline $\begin{array}{l}\text { Overlap-Syndrom } \\
\text { (Mischbild von akuter } \\
\text { und chronischer GVHD) }\end{array}$ & kein Zeitlimit & ja & ja \\
\hline
\end{tabular}

seits wird durch Beschleunigung der Apoptose von aktivierten Donor-T-Zellen die GvHD gedämpft $[6,10]$. Die Effektorphase wird maßgeblich durch zytotoxische T-Zellen und NK-Zellen bestimmt. Es kommt zur Fas-Fas-Ligand- und Perforin-Granzym-B-mediierten Schädigung der Hepatozyten, Darmepithelien und Keratinozyten. Für die Manifestation des Krankheitsbilds ist das von aktivierten T-Lymphozyten freigesetzte TNF- $\alpha$, das zur Freisetzung weiterer Zytokine, wie IL-1, IL-6, IL-12 aus zytotoxischen T-Zellen und NK-Zellen führt, von maßgeblicher Bedeutung $[6,10,11]$.

In der aGvHD können 3 Phasen unterschieden werden: Aktivierung antigenpräsentierender Zellen des Empfängers, Aktivierung von Donor-T-Zellen und Effektorphase.

\section{Chronische GVHD}

Die cGvHD ist eine Multiorganerkrankung, die Ähnlichkeit mit Autoimmunerkrankungen (Sklerodermie, Lupus erythematodes, Sjögren-Syndrom, primär biliäre Zirrhose) aufweist. Die Pathophysiologie ist komplex und weit weniger verstanden als die der aGvHD. Aus experimentellen Modellen wurden verschiedene Hypothesen entwickelt, wie die cGvHD entsteht. Eine davon besagt, dass durch die Konditionierung und die aGvHD-hervorgerufene Schädigung des Thymusgewebes die T-Zell-Selektion im Thymus nicht mehr ausreichend funktioniert und so autoreaktive $\mathrm{CD}^{+}$-T-Zellen das Thymusgewebe verlassen können $[12,13]$. Auch gibt es Hinweise darauf, dass B-Zellen in der Entstehung der cGvHD eine Rolle spielen [14]. Bei einer geschlechtsunterschiedlichen Transplantation werden Alloantikörper gegen Y-Chromosom-assoziierte MiHA (H-YAntikörper) gebildet, die mit dem Auftreten der cGvHD korrelieren.

Bei Sklerodermie-Patienten und Patienten mit cGvHD, nicht aber bei Patienten ohne cGvHD wurden stimulierende Antikörper gegen den PDGF-Rezeptor (PDGF: platelet-derived growth factor) gefunden, die in vitro die Bildung von Kollagen in humanen Fibroblasten induzieren kön-

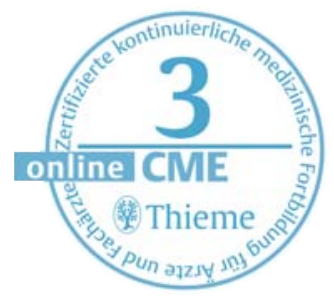




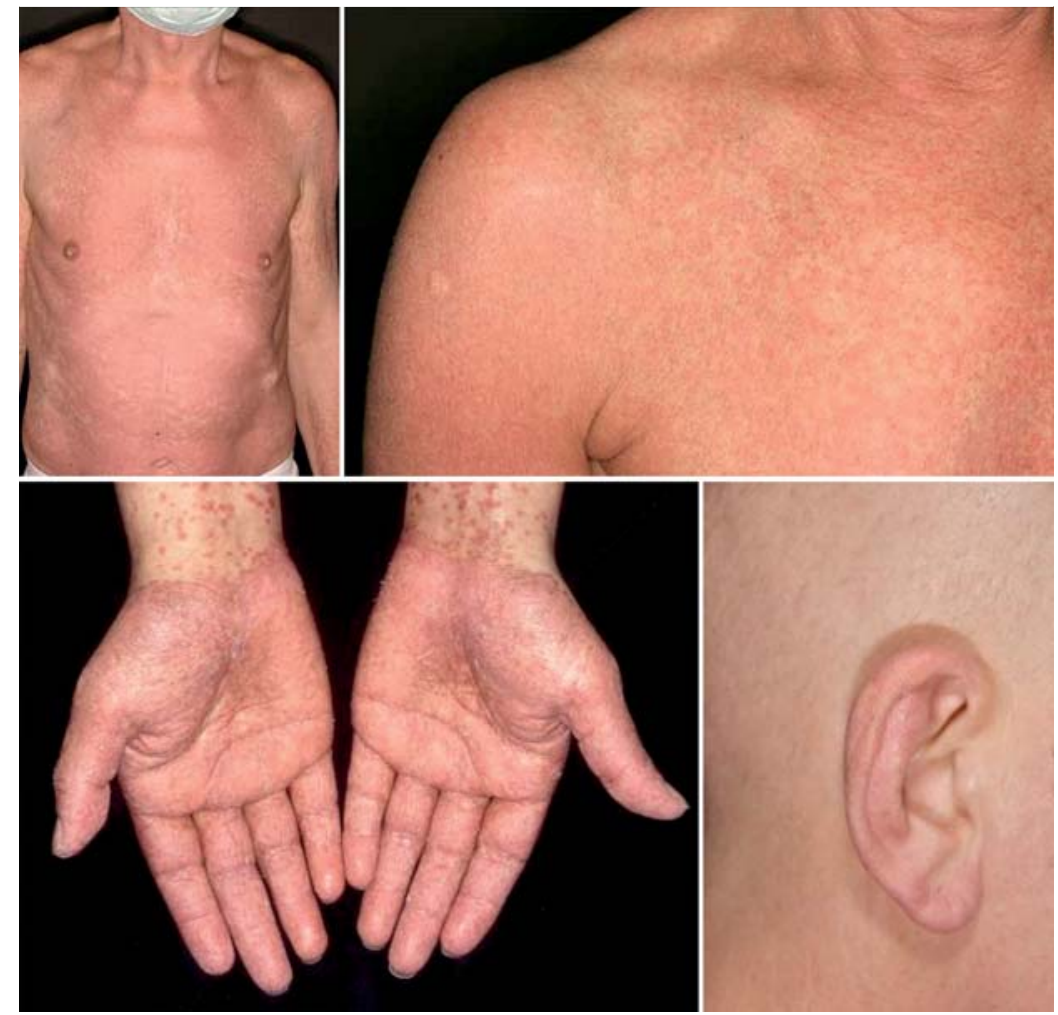

Abb. 1 Akute GvHD mit konfluierendem makulösem Exanthem (oben), Befall der Palmae und Ohren (unten).

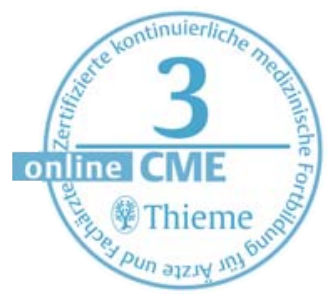

nen [15]. Es konnte bisher aber nicht gezeigt werden, dass die Autoantikörper direkt für die Entwicklung der cGvHD verantwortlich sind. Möglicherweise stellen sie eher einen Marker für das Vorhandensein antigenspezifischer B-Zellen dar, die über andere Mechanismen an der Entwicklung der cGvHD beteiligt sind. Jedenfalls ist ein höherer Anteil von B-Zellen im Transplantat mit einem erhöhten Risiko für das Auftreten einer cGvHD verknüpft.

Ein weiterer Hinweis auf die Bedeutung von BZellen ist die Beobachtung, dass die cGvHD auf eine Therapie mit dem Anti-CD20-Antikörper Rituximab anspricht. Hier zeigen sich organabhängig unterschiedliche Ansprechraten (Haut, muskuloskeletale Manifestation, Mundschleimhaut $71-100 \%$ ) [16]. Des Weiteren scheint die Balancierung der Immunreaktion durch regulatorische T-Tellen (Tregs) bei der GvHD gestört zu sein. Natürliche Tregs bilden einen Anteil von 5$10 \%$ in der T-Zell-Population. Sie sind positiv für CD4 und CD25, exprimieren hohe Level an FOXP3 und sind negativ oder nur schwach positiv für CD127 (IL-7-Rezeptor alpha). Es wurde beobachtet, dass bei Patienten mit einer GvHD der relative Anteil von Tregs in der Kolonmukosa im Vergleich zu Patienten mit einer CMV-Kolitis (CMV: Zytomegalievirus) oder Divertikulitis vermindert war [17]. Im murinen Modell waren Tregs in der Lage, die Empfänger vor einer letalen aGvHD zu schützen, wenn sie 1:1 mit Effektor-T-Zellen appliziert wurden $[18,19]$. Ein erhöhter Anteil von Tregs im Transplantat war assoziiert mit einem geringeren Risiko, eine cGvHD zu entwickeln, was ebenfalls auf die mögliche Bedeutung der Tregs für die
cGvHD hinweist. Allerdings ist die Datenlage dazu bisher nicht eindeutig [12].

Die cGvHD ist eine Multiorganerkrankung, die Ähnlichkeit mit Autoimmunerkrankungen aufweist. Die Pathophysiologie der cGvHD ist komplex und weit weniger verstanden als die der aGvHD.

\section{Klinik und Diagnostik der GvHD \\ $\nabla$}

\section{Akute GvHD}

Nach myeloablativer Konditionierung setzt die aGvHD etwa 2-4 Wochen nach SZT ein. Wenn ein Schema mit reduzierter Konditionierungsintensität angewendet wird, kann die aGvHD verspätet nach 2-3 Monaten auftreten und sich als verzögerte aGvHD oder Overlap-Syndrom manifestieren [6]. Die aGvHD manifestiert sich vorwiegend an Haut, Gastrointestinaltrakt und Leber.

Gastrointestinale und hepatische Symptome: Die gastrointestinale aGvHD manifestiert sich in Diarrhöen, wenn der obere Gastrointestinaltrakt betroffen ist auch mit Anorexie, Übelkeit und Erbrechen. Die Diarrhöen können massiv sein mit Stuhlmengen von $>2 \mathrm{l} / \mathrm{d}$ und bei schweren Verläufen mit krampfartigen abdominellen Schmerzen und Ileus einhergehen. Bei Erosion/ Ulzeration der Mukosa kommt es zum Auftreten blutiger Stühle, was ein prognostisch schlechtes Zeichen darstellt [6]. Die akute hepatische GvHD manifestiert sich als Hyperbilirubinämie und Anstieg der alkalischen Phosphatase (AP) und einem geringen Anstieg der Transaminasen.

Kutane Symptome ( $\bullet$ Abb. 1): Die Haut ist das am häufigsten betroffene Organ. Relativ charakteristische Zeichen sind palmoplantare Erytheme und lividrote Erytheme der Ohrmuscheln sowie perifollikuläre Erytheme und Papeln. Die palmoplantaren Erytheme können von Ödemen und starker Druckschmerzhaftigkeit begleitet sein. Die aGvHD tritt häufig zuerst an den Ohren, Wangen, Händen und Füßen auf und generalisiert je nach Schwere der Reaktion unter dem Bild eines in der Regel symmetrischen morbilliformen oder makulopapulösen teils hämorrhagischen, juckenden oder schmerzhaften Exanthems, das bei zunehmenden Apoptosen der Keratinozyten zur Formation von Bullae führen kann und in maximaler Ausprägung dem Bild der toxischen epidermalen Nekrolyse gleicht. Die Kopfhaut ist in der Regel ausgespart. Im Rahmen der aGvHD kann es auch zu einer schweren erosiven Stomatitis kommen.

Nach myeloablativer Konditionierung setzt die aGvHD etwa 2-4 Wochen nach SZT ein, nach reduzierter Konditionierungsintensität kann sie verzögert nach 2 - 3 Monaten auftreten. Die aGvHD manifestert sich hauptsächlich an Haut, Darm und Leber. 


\section{Schweregradeinteilung}

Es wurden verschiedene Gradingsysteme entwickelt. Das erste detaillierte Grading geht auf Glucksberg zurück [21]. Der Befall der Organe Haut, Leber und Darm (Gastrointestinaltrakt) wird in Stadien 1-4 graduiert und abhängig vom Befall der einzelnen Organe ein Gesamtschweregrad I-IV ermittelt. In der Konsensuskonferenz von 1994 wurde diese Einteilung dahingehend modifiziert, dass persistierende Übelkeit mit histologischer Evidenz auf eine GvHD in das Stadium 1 der aGvHD des Gastrointestinaltrakts aufgenommen wurde ( $\bullet$ Tab. 3) [22]. Der IBMTR-Severity-Index betrachtet ebenfalls die 3 Organe und erstellt aus dem Stadium eine Kategorie A-D. Die schwere aGvHD ist ein lebensbedrohliches Krankheitsbild. Die Überlebensprognose für das erste Jahr nach Transplantation beträgt im Stadium III 30\% und im Stadium IV $<10 \%$ [23].

\section{Chronische GvHD}

Bei Patienten, die aufgrund einer akuten oder chronischen myeloischen Leukämie, aplastischen Anämie oder lymphoblastischen Leukämie transplantiert wurden und länger als 2 Jahre rezidivfrei blieben, ist auch Jahre nach Transplantation die Mortalität im Vergleich zur übrigen Bevölkerung erhöht. Die Haupttodesursache bei diesen Patienten ist die cGvHD, die damit die schwerwiegendste Langzeitkomplikation der SZT darstellt [24]. Dabei kann die cGVHD die direkte (z. B. pulmonale GvHD, Bronchiolitis obliterans) oder indirekte Todesursache sein. Letztere wird aufgrund der durch die Erkrankung selbst und/oder aufgrund der zu ihrer Behandlung eingesetzten immunsuppressiven Therapie hervorgerufenen Immundefizienz, die eine Basis für schwere Infektionen bildet, verursacht. Aufgrund der Zunahme der Transplantationszahlen und der Transplantationen mit Vorliegen von Risikofaktoren für das Auftreten einer cGvHD, der Abnahme der Frühmortalität infolge weiterentwickelter Konditionierungsschemata und verbesserten Behandlungsmöglichkeiten nach der Transplantation (z.B. antiinfektive Therapien) nimmt die Anzahl der Patienten, die potenziell eine cGvHD entwickeln können, zu [25].

Die cGvHD ist die schwerwiegendste Langzeitkomplikation der SZT.

Risikofaktoren für das Auftreten einer cGvHD stellen die HLA-Disparität zwischen Spender und Empfänger, eine vorausgegangene aGvHD, ein höheres Alter von Spender oder Empfänger, die Konstellation weiblicher Spender und männlicher Empfänger und die Verwendung von Blutstammzellen als Transplantatquelle dar [25]. Die cGvHD kann sich direkt aus der aGvHD (progressive Disease), nach vorausgegangener aGvHD mit symptomfreiem Intervall (quiescent Onset) und
Tab. 3 Grading der akuten GvHD entsprechend Konsensuskonferenz 1994 [nach 22].

\begin{tabular}{|c|c|c|c|}
\hline Stadium & Haut & $\begin{array}{l}\text { Leber } \\
\text { [Bilirubin } \\
\text { mg/dl] }\end{array}$ & $\begin{array}{l}\text { Darm } \\
\text { [Stuhlmenge } \\
\text { ml/Tag] }\end{array}$ \\
\hline 1 & $\begin{array}{l}\text { makulopapulöses } \\
\text { Exanthem < } 25 \% \text { KOF }\end{array}$ & $2-<3$ & $\begin{array}{l}\text { > } 500 \text { oder persistente } \\
\text { Übelkeit mit histologi- } \\
\text { scher Evidenz für } \\
\text { GvHD in Magen oder } \\
\text { Duodenum }\end{array}$ \\
\hline 2 & $\begin{array}{l}\text { makulopapulöses } \\
\text { Exanthem } 25-50 \% \\
\text { KOF }\end{array}$ & $3-<6$ & $>1000$ \\
\hline 3 & $\begin{array}{l}\text { makulopapulöses } \\
\text { Exanthem > 50\% } \\
\text { KOF/Erythrodermie }\end{array}$ & $6-<15$ & $>1500$ \\
\hline 4 & $\begin{array}{l}\text { Erythrodermie mit } \\
\text { Blasen/Epidermolyse }\end{array}$ & $\geq 15$ & $\begin{array}{l}\text { schwere abdominelle } \\
\text { Schmerzen + / - Ileus }\end{array}$ \\
\hline Schweregrad & Stadium & Stadium & Stadium \\
\hline I & $1-2$ & & \\
\hline II & 3 und/oder & 1 und/oder & 1 \\
\hline III & $1-3$ & $2-3$ und/oder & $2-4$ \\
\hline $\mathrm{IV}^{*}$ & 4 und/oder & 4 & \\
\hline
\end{tabular}

KOF: Körperoberfläche

* Grad IV kann auch bei niedrigeren Stadien der Organmanifestation erreicht werden, wenn der Allgemeinzustand gemessen am Performance-Status erheblich reduziert ist.

ohne vorausgegangene aGvhD (de novo Onset) entwickeln. Eine progressive Disease stellt einen Risikofaktor hinsichtlich transplantationsassoziierter Mortalität dar.

Risikofaktoren für das Auftreten einer cGvHD sind HLADisparität zwischen Spender und Empfänger, eine vorausgegangene aGvHD, höheres Alter von Spender oder Empfänger, die Konstellation weiblicher Spender und männlicher Empfänger und die Verwendung von Blutstammzellen als Transplantatquelle.

Die cGvHD tritt bei 30-70\% der Patienten, die die Transplantation mehr als 100 Tage überleben, im Median 4-6 Monate nach der SZT auf [26]. Bei 5$10 \%$ der Patienten wird die Erkrankung aber erst später als $1 \mathrm{Jahr}$ nach SZT festgestellt. Bei etwa der Hälfte der Patienten sind 3 oder mehr Organe betroffen, im Median ist eine immunsuppressive Therapie über 2-3 Jahre erforderlich. Allerdings benötigen etwa $15 \%$ der Patienten eine Langzeitimmunsuppression über 7 und mehr Jahre nach Diagnosestellung der cGvHD [26].

Die cGvHD ist eine Multiorganerkrankung, das am häufigsten betroffene Organ ist die Haut mit ihren angrenzenden Schleimhäuten, weitere häufig betroffene Organe sind Augen, Leber und Gastrointestinaltrakt ( $\odot$ Tab.4) [26]. Die cGvHD führt zu einer erheblichen Beeinträchtigung der Lebensqualität [27].

Die cGvHD ist eine Multiorganerkrankung, die eine meist über mehrere Jahre andauernde immunsuppressive Therapie erfordert.

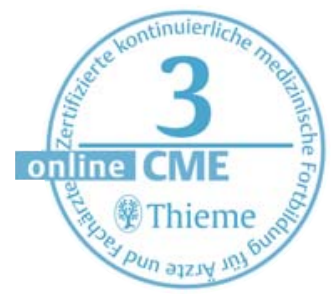


Tab. 4 Häufigkeit des Organbefalls der chronischen GvHD [nach 26].

\begin{tabular}{|l|l|}
\hline Mundschleimhaut & $89 \%$ \\
\hline Haut & $81 \%$ \\
\hline Gastrointestinaltrakt & $48 \%$ \\
\hline Leber & $47 \%$ \\
\hline Augen & $47 \%$ \\
\hline myofaszialer Befall & $16 \%$ \\
\hline Gelenke & $15 \%$ \\
\hline Lunge & $14 \%$ \\
\hline Genitale (Penis, Vagina) & $10 \%$ \\
\hline Ösophagus & $5 \%$ \\
\hline
\end{tabular}

Haut ( $\odot$ Abb. 2, Abb. 3): Die cGvHD der Haut wird meist in die 2 großen Kategorien lichenoide und sklerodermiforme GvHD eingeteilt. Die cGvHD kann sich allerdings mit unspezifischen und diskreten Zeichen ankündigen. Dazu gehören das Auftreten einer Xerosis cutis, teils unter dem Bild einer Ichthyose, perifollikuläre Erytheme, die bei stärkerer Infiltration Keratosis-pilaris-artig imponieren, Pityriasis-rosea-artige Läsionen, psoriasiforme Plaques und anuläre Erytheme [28].

Die lichenoide cGvHD manifestiert sich mit Lichen-planus-artigen Papeln und Plaques. Diese können symmetrisch angeordnet sein, jedoch auch follikulozentrisch, lineär sein oder einem Dermatom folgen. Die Manifestation der lichenoiden und sklerodermiformen Hauptformen kann sich überlagern. Auch finden sich nicht immer typische Lichen-planus-artige Efforeszenzen, sondern eher makulopapulöse Exantheme oder erythematöse, schuppende Plaques, die sich bis zur Erythrodermie weiterentwickeln können. Palmoplantar können ebenfalls schuppende infiltrierte Erytheme auftreten, die durchaus einem
Handekzem (dyshidrosiform oder hyperkeratotisch) ähnlich sehen und mit teils starken Schmerzen einhergehen können. An den Händen können Dermatomyositis-artige livide Erytheme über den Fingergelenken vorkommen.

Die sklerodermiforme cGvHD manifestiert sich klinisch mit einer oberflächlichen und/oder tiefen Sklerose ( Abb. 4). Die oberflächliche sklerosierende Form zeigt sich unter dem Bild eines Lichen sclerosus oder einer Morphea. Bei tiefer Sklerose mit Beteiligung der Subkutis und der Faszien zeigt sich eine matratzenartige oder streifenförmige (grooving) Einziehung der Haut, und es entwickelt sich eine zunehmende Einschränkung der Beweglichkeit der Patienten, insbesondere, wenn die Sklerose gelenkübergreifend ist. Die Sklerose kann das gesamte Integument befallen, das klinische Bild ist dem einer schweren systemischen Sklerodermie ähnlich. Nicht selten ist auch das Capillitium betroffen, was mit einer vernarbenden Alopezie einhergehen kann. Infolge der Schädigung der epidermodermalen Junktionszone kommt es zu dermaler Ansammlung von Melanophagen, was zu persistierenden postinflammatorischen Hyperpigmentierungen führt. Komplizierend treten subepidermale Blasen und Lymphzysten auf, die rupturieren und sich zu schlecht heilenden Ulzera weiterentwickeln können. Diagnostisch für eine cGvHD ist auch eine Poikilodermie. Die Nägel sind häufig betroffen. Je nach Schweregrad der Schädigung entwickelt sich eine Nageldystrophie mit Längsriffelung des Nagels, Verdünnung der Nagelplatte bis hin zum völligen Nagelverlust. Charakteristisch ist ein Pterygium. Periungual können Teleangieektasien auftreten.
Abb. 2 Chronische lichenoide GvHD: Befall der Palmae (oben) mit anulären lichenoiden Papeln (links) und hyperkeratotischen infiltrierten Plaques (rechts), Manifestation an den Nägeln mit beginnendem Nagelverlust (unten links) und frühe Keratosis-pilaris-artige Veränderungen (unten rechts).
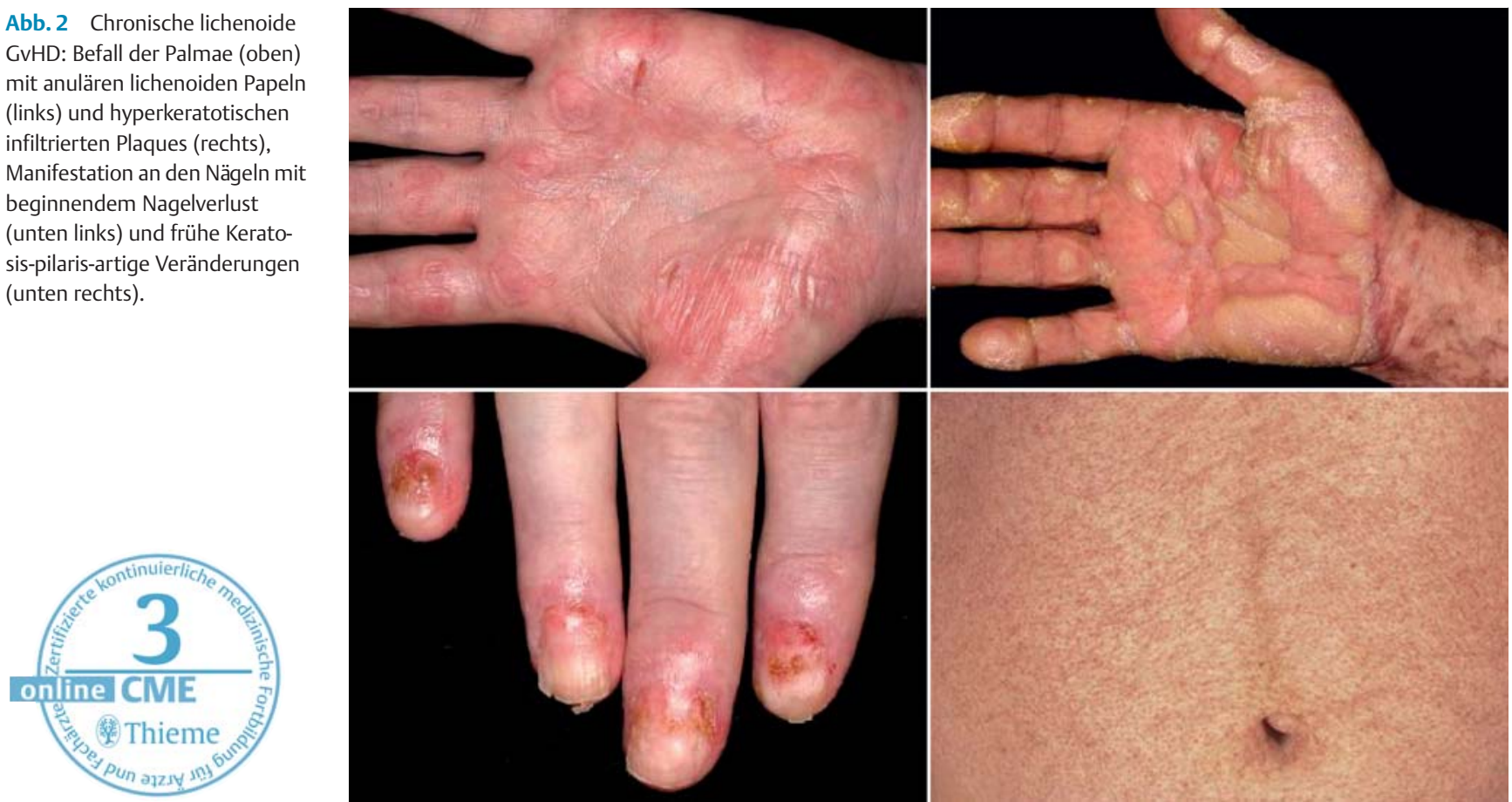
Mundschleimhaut: Die cGvHD an der Mundschleimhaut hat klinisch Ähnlichkeit mit dem Lichen mucosae. An der Wangenschleimhaut und den Lippen findet sich eine netzartige weißliche Zeichnung sowie flächige Erytheme. Es können schmerzhafte Erosionen und Pseudomembranen auftreten, die den Patienten in der Nahrungsaufnahme erheblich einschränken. Des Weiteren gehören zum Spektrum der chronischen Mundschleimhaut-GvHD eine Gingivitis und Glossitis, teils mit Atrophie der Zungenpapillen, hyperkeratotische/leukoplakische Bezirke, die eine differenzialdiagnostische Abgrenzung zu einem Plattenepithelkarzinom erfordern, für dessen Entwicklung die erosive cGvHD prädisponiert. Häufig besteht eine Sicca-Symptomatik, in deren Gefolge sich Karies ausbildet. Bei perioraler Sklerose kann es zu einer starken Einschränkung der Mundöffnung kommen. Insbesondere die erosiven Läsionen prädisponieren für eine Superinfektion (Herpesviren, Kandidose) [25].

Genitalschleimhaut: Die vaginale cGvHD entwickelt sich durchschnittlich 10 Monate nach SZT [28]. Die Symptome sind ähnlich wie an den anderen Schleimhäuten mit Trockenheit und Verdickung der Schleimhaut, auftretenden Erosionen oder Ulzera, Strikturen bis hin zur Obliteration des Introitus. Bei Männern kann eine entzündliche Phimose auftreten [25].

Augen: Die Sicca-Symptomatik betrifft häufig auch die Tränendrüsen, sodass sich eine Keratoconjunctivitis sicca entwickelt. Die Patienten

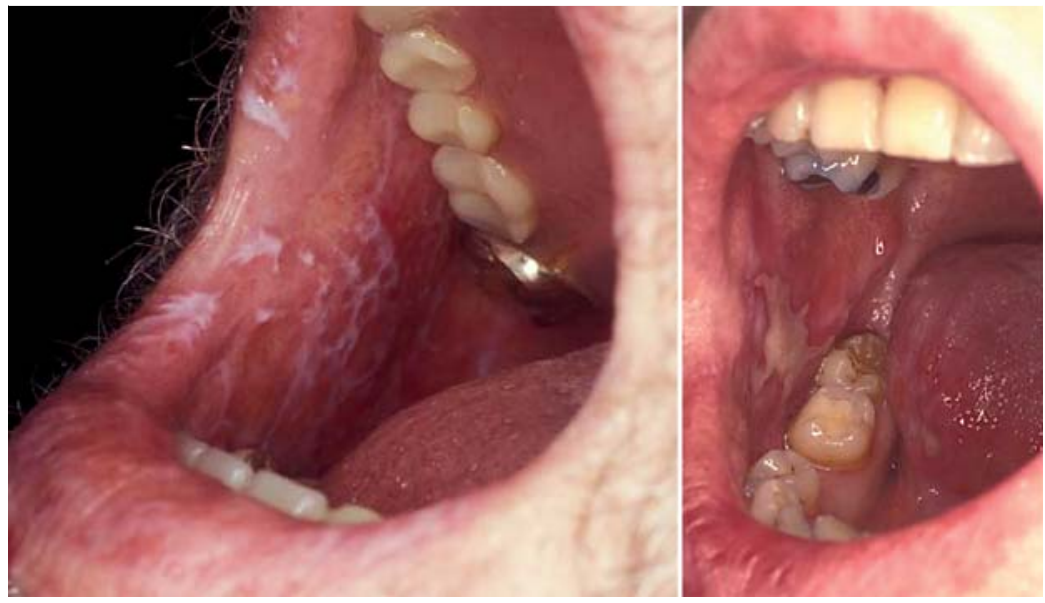

Abb. 3 Mundschleimhautveränderungen bei chronischer GvHD: netzartige Schleimhautzeichnung (links) und Pseudomembranen (rechts).

klagen über trockene Augen, Fremdkörpergefühl, Brennen, Schmerzen und Verschwommensehen, eine Fotophobie kommt dazu. Komplizierend kann es zu Hornhauterosionen und -ulzerationen, Sekundärinfektionen, Vaskularisierung der Hornhaut und Narbenektropium kommen [25].

Lungen: Die cGvHD der Lunge ist eine potenziell lebensbedrohliche Erkrankung. Es entsteht eine Bronchiolitis obliterans, die in eine respiratorische Insuffizienz führen kann. Die Patienten haben trockenen Husten und zunehmende Kurzatmigkeit [25].
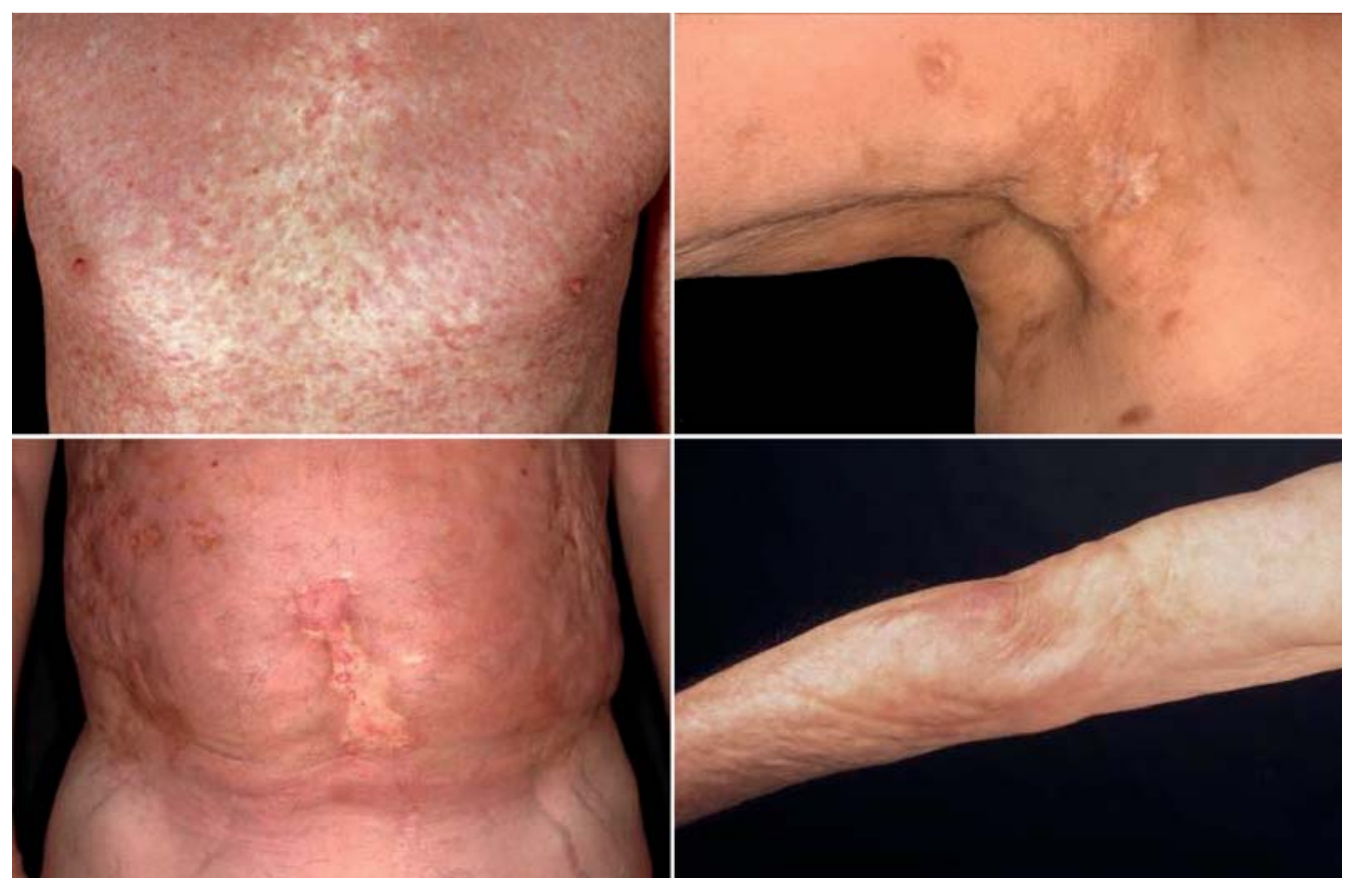

Abb. 4 Sklerodermiforme chronische GvHD: oberflächliche Sklerose mit Teleangiektasien und diskreter Hyperpigmentierung (oben links), Lichen-sklerosus-artige Sklerose (oben rechts), tiefe Sklerose mit matratzenartiger Einziehungen seitlich am Abdomen und zentral abgeheiltem Ulkus (unten links) und streifenförmiger Einziehung bei Fasziensklerose (unten rechts).

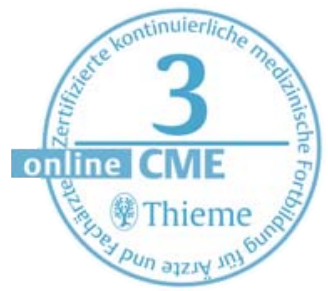


Tab. 5 Symptome und klinische Merkmale der chronischen GvHD nach NIH-Konsensus [nach 20].

\begin{tabular}{|c|c|c|c|c|}
\hline & Diagnostische Zeichen ${ }^{1}$ & $\begin{array}{l}\text { Charakteristische } \\
\text { Zeichen }^{2}\end{array}$ & Andere Symptome ${ }^{3}$ & $\begin{array}{l}\text { Gemeinsame Symptome/ } \\
\text { Merkmale }^{4}\end{array}$ \\
\hline Haut & $\begin{array}{l}\text { - Poikilodermie } \\
\text { - Lichen-sklerosus-artige } \\
\text { Veränderungen } \\
\text { - Lichen-planus-artige } \\
\text { Veränderungen } \\
\text { - sklerodermiforme } \\
\text { Veränderungen }\end{array}$ & $\begin{array}{l}\text { - Depigmentierung } \\
\text { - neu aufgetretene vernar- } \\
\text { bende oder nicht vernar- } \\
\text { bende Alopezie (nach } \\
\text { Abklingen der Radio- } \\
\text { Chemotherapie-Toxizität) } \\
\text { - Schuppung, papulo- } \\
\text { squamöse Läsionen an } \\
\text { der Kopfhaut }\end{array}$ & $\begin{array}{l}\text { - reduzierte Schweiß- } \\
\text { sekretion } \\
\text { - Ichthyose } \\
\text { - Keratosis-pilaris-artige } \\
\text { Veränderungen } \\
\text { - Hypopigmentierungen } \\
\text { - Hyperpigmentierung } \\
\text { - verfrühtes Ergrauen der } \\
\text { Haare } \\
\text { - Verdünnung der Haare }\end{array}$ & $\begin{array}{l}\text { - „Erytheme“ } \\
\text { - makulokapulöses } \\
\text { Exanthem } \\
\text { - Pruritus }\end{array}$ \\
\hline Nägel & & $\begin{array}{l}\text { - Nageldystrophie } \\
\text { - Longitudinalriffelung, } \\
\text { Splitterung der Nägel } \\
\text { - Onycholyse } \\
\text { - Pterygium } \\
\text { - Nagelverlust* }\end{array}$ & & \\
\hline $\begin{array}{l}\text { Mundschleim- } \\
\text { haut }\end{array}$ & $\begin{array}{l}\text { - eingeschränkte Mund- } \\
\text { öffnung infolge Sklerose } \\
\text { - Lichen-mucosae-artige } \\
\text { Veränderungen } \\
\text { - hyperkeratotische Plaques }\end{array}$ & $\begin{array}{l}\text { - Xerostomie } \\
\text { - Mukozele } \\
\text { - Pseudomembranen* } \\
\text { - Schleimhautatropie } \\
\text { - Ulzera* }\end{array}$ & & $\begin{array}{l}\text { - Gingivitis } \\
\text { - Mukositis } \\
\text { - Erythem } \\
\text { - Schmerzen }\end{array}$ \\
\hline Augen & & $\begin{array}{l}\text { - Neues Trockenheitsgefühl, } \\
\text { Fremdkörpergefühl („Sand } \\
\text { im Auge“) oder Augen- } \\
\text { schmerzen } \\
\text { - vernarbende Konjunktivitis } \\
\text { - Keratoconjunctivitis sicca } \\
\text { - konfluierende Areale einer } \\
\text { punktförmigen Kerato- } \\
\text { pathie }\end{array}$ & $\begin{array}{l}\text { - Fotophobie } \\
\text { - periorbitale Hyperpig- } \\
\text { mentierung } \\
\text { - Blepharitis (Erythem der } \\
\text { Augenlider mit Ödem) }\end{array}$ & \\
\hline Genitalien & $\begin{array}{l}\text { - Lichen-planus-artige } \\
\text { Veränderungen } \\
\text { - vaginale Vernarbung oder } \\
\text { Stenosen }\end{array}$ & $\begin{array}{l}\text { - Erosionen* } \\
\text { - Fissuren* } \\
\text { - Ulzerationen* }\end{array}$ & & \\
\hline $\begin{array}{l}\text { Gastrointestinal- } \\
\text { trakt }\end{array}$ & $\begin{array}{l}\text { - ösophageales Netz } \\
\text { - Strikturen oder Stenose im } \\
\text { oberen oder mittleren } \\
\text { Drittel des Ösophagus* }\end{array}$ & & $\begin{array}{l}\text { - exokrine Pankreas- } \\
\text { insuffizienz }\end{array}$ & $\begin{array}{l}\text { - Anorexie } \\
\text { - Übelkeit } \\
\text { - Erbrechen } \\
\text { - Diarrhö } \\
\text { - Gewichtsverlust } \\
\text { - Wachstumsstörung } \\
\text { (Kinder) }\end{array}$ \\
\hline Leber & & & & $\begin{array}{l}\text {-Gesamtbilirubin, } \\
\text { AP }>2 \text {-fach des oberen } \\
\text { Normwerts* } \\
\text { - GOT oder GPT > 2-fach des } \\
\text { oberen Normwerts* }\end{array}$ \\
\hline Lunge & $\begin{array}{l}\text { - mittels Lungenbiopsie } \\
\text { diagnostizierte Bronchio- } \\
\text { litis obliterans }\end{array}$ & $\begin{array}{l}\text { - mittels Lungenfunktions- } \\
\text { test und radiologisch } \\
\text { diagnostizierte Bronchio- } \\
\text { litis obliterans }\end{array}$ & & $\begin{array}{l}\text { - Bronchiolitis obliterans } \\
\text { mit organisierender } \\
\text { Pneumonie }\end{array}$ \\
\hline $\begin{array}{l}\text { Muskeln, Faszien } \\
\text { und Gelenke }\end{array}$ & $\begin{array}{l}\text { - Fasziitis } \\
\text { - Gelenkversteifungen oder } \\
\text { Kontrakturen infolge von } \\
\text { Sklerose }\end{array}$ & - Myositis oder Polymyositis & $\begin{array}{l}\text { - Ödeme } \\
\text { - Muskelkrämpfe } \\
\text { - Arthralgien oder Arthritis }\end{array}$ & \\
\hline $\begin{array}{l}\text { hämatopoeti- } \\
\text { sches System und } \\
\text { Immunsystem }\end{array}$ & & & & $\begin{array}{l}\text { - Thrombozytopenie } \\
\text { - Eosinophilie } \\
\text { - Lymphopenie } \\
\text { - Hypo- oder Hypergamma- } \\
\text { globulinämie } \\
\text { - Autoantikörper (auto- } \\
\text { immunhämolytische } \\
\text { Anämie, idiopathische } \\
\text { thrombozytopenische } \\
\text { Purpura) }\end{array}$ \\
\hline
\end{tabular}


Tab. 5 (Fortsetzung)

\begin{tabular}{|c|c|c|c|c|}
\hline & Diagnostische Zeichen ${ }^{1}$ & $\begin{array}{l}\text { Charakteristische } \\
\text { Zeichen }^{2}\end{array}$ & Andere Symptome ${ }^{3}$ & $\begin{array}{l}\text { Gemeinsame Symptome/ } \\
\text { Merkmale }^{4}\end{array}$ \\
\hline andere Organe & & & & $\begin{array}{l}\text { - Perikard-oder Pleuraerguss } \\
\text { - Aszites } \\
\text { - periphere Neuropathie } \\
\text { - nephrotisches Syndrom } \\
\text { - Myasthenia gravis } \\
\text { - kardiale Reizleitungs- } \\
\text { störungen oder Kardio- } \\
\text { myopathie }\end{array}$ \\
\hline
\end{tabular}

${ }^{1}$ Vorhandensein berechtigt zu Diagnosestellung einer cGvHD

${ }^{2}$ Symptome/Merkmale kommen bei cGvHD vor, alleiniges Vorhandensein dieser Symptome nicht ausreichend für Diagnosestellung

${ }^{3}$ Symptome/Merkmale können als cGvHD-Manifestation betrachtet werden, sofern Diagnose der cGvHD gesichert

${ }^{4}$ kommen bei akuter und chronischer GvHD vor

*differenzialdiagnostischer Ausschluss einer Infektion, Medikamentennebenwirkung oder anderer Ursachen erforderlich

Gastrointestinaltrakt und Leber: cGvHD des Gastrointestinaltrakts kann sich in Übelkeit, Erbrechen, Diarrhö, abdominellen Krämpfen und bei Beteiligung des oberen Gastrointestinaltrakts in Dysphagie äußern. Je nach Schwere kann es zum Gewichtsverlust und Wasting-Syndrom kommen. Die Symptomatik muss von anderen Ursachen, wie Infektionen (z.B. CMV-Kolitis, pseudomembranöse Kolitis durch Clostridium difficile) oder Medikamentennebenwirkungen, abgegrenzt werden. Die chronische Leber-GvHD führt zu einer Cholestase mit Hyperbilirubinämie, selten zu einer akuten Hepatitis [25].

Hämatopoetisches System: Eine Thrombopenie von $<100000 / \mu l$ bei Diagnosestellung stellt ein schlechtes prognostisches Zeichen dar. Eine post transplantationem auftretende Zytopenie ist erst dann als Manifestation einer cGvHD zu bewerten, wenn andere Ursachen ausgeschlossen sind (Rezidiv der Grunderkrankung, Medikamententoxizität, Folgen der Konditionierung, virale Infektionen, autoimmunologisch bedingte Zytopenien) [25].

Nervensystem: Die Beteiligung des Nervensystems ist selten und vielgestaltig und kann sich unter dem Bild einer Myasthenia gravis äußern, mit Myositis und Polyneuropathie einhergehen. Differenzialdiagnostisch müssen andere, z.B. medikamentös-toxische Ursachen abgegrenzt werden [25].

\section{Diagnosestellung}

Die Arbeitsgruppe des NIH hat die im Rahmen einer GvHD in den verschiedenen Organen auftretenden klinischen Symptome hinsichtlich ihrer diagnostischen Bedeutung bewertet [20] ( Tab. 5).

Es werden grundsätzlich diagnostische Zeichen von charakteristischen (aber nicht diagnostischen) Symptomen unterschieden. Diagnostische Zeichen erlauben die Diagnosestellung einer cGvHD, während charakteristische Zeichen zwar auf das Vorliegen einer cGvHD hinweisen, diese aber für sich allein nicht beweisen und deshalb einer Bestätigung bedürfen. Des Weiteren wurden Symptome definiert, die sowohl bei der cGvHD als auch bei der aGvHD (gemeinsame Zeichen) vorkommen. Unter anderen Zeichen wurden seltene oder unspezifische Symptome benannt, die nicht zur Diagnose der cGvHD herangezogen werden können, aber als Teil der cGvHD-Manifestation/ Symptomatik betrachtet werden können, wenn die Diagnose gesichert ist.

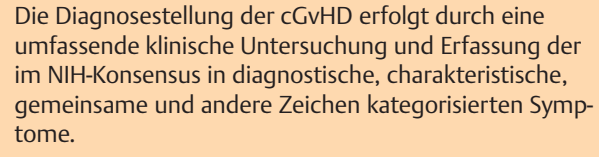
im NIH-Konsensus in diagnostische, charakteristische, gemeinsame und andere Zeichen kategorisierten Symptome.

Die cGvHD-Diagnostik auf Basis der NIH-Empfehlungen [20] erfordert eine vollständige Untersuchung des Patienten im Hinblick auf das evtl. Vorhandensein von Symptomen in allen möglichen Manifestationsorganen der cGvHD. Die Diagnose einer cGvHD kann gestellt werden, wenn mindestens 1 diagnostisches Zeichen vorhanden ist. Weist der Patient nur charakteristische Zeichen auf, kann die Diagnose einer cGvHD dann gestellt werden, wenn in demselben oder einem anderen Organ die Diagnose histologisch, laborchemisch oder radiologisch bestätigt wird. An der Haut und der Mundschleimhaut können die wichtigen diagnostischen und charakteristischen Symptome gefunden werden. Die dermatologische Untersuchung stellt, da Haut und Mundschleimhaut somit „diagnostische“ Organe sind, eine wesentliche Säule für die Diagnosestellung der cGvHD dar.

Die Haut ist ein „diagnostisches“ Organ, die dermatologische Untersuchung stellt daher eine wesentliche Säule für die Diagnosestellung der cGvHD dar.

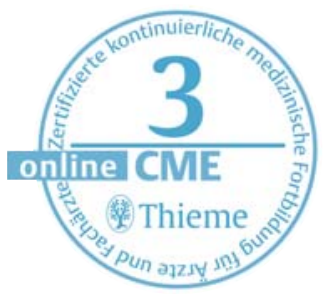


Tab. 6 Organscoring der CGvHD nach NIH-Empfehlungen: Haut und Mundschleimhaut [nach 20].

\begin{tabular}{|l|l|}
\hline & \multicolumn{2}{l}{ Score } \\
\hline Haut & 0 \\
\hline keine Symptome & 1 \\
\hline$<18 \%$ der Körperoberfläche betroffen, aber keine Sklerose & 2 \\
\hline $\begin{array}{l}19-50 \% \text { der Körperoberfläche betroffen ODER Vorhandensein oberflächlicher } \\
\text { Sklerose }\end{array}$ & 3 \\
\hline \begin{tabular}{l} 
> 50\% der Körperoberfläche betroffen ODER Vorhandensein tiefer Sklerose \\
\hline ODER Ulzeration, eingeschränkte Mobilität ODER schwerer Pruritus
\end{tabular} & \\
\hline Mundschleimhaut & 1 \\
\hline keine Symptome & 2 \\
\hline milde Symptome ohne Beeinträchtigung der Nahrungsaufnahme & 3 \\
\hline moderate Symptome mit parzieller Einschränkung der Nahrungsaufnahme & \\
\hline schwere Symptome mit erheblicher Einschränkung der Nahrungsaufnahme & \\
\hline
\end{tabular}

\section{Schweregradeinteilung}

Die seit 1980 benutzte Schwergradeinteilung unterscheidet zwischen einer limitierten und einer ausgedehnten cGvHD. Die NIH-Arbeitsgruppe hat ein neues Scoring-System erarbeitet, das nunmehr angewendet werden soll [20]. Zu unterscheiden sind der Schweregrad bezogen auf das einzelne Organ und der Gesamtschweregrad der cGvHD. Letzterer ergibt sich aus der Anzahl der betroffenen Organe und aus dem Schweregrad der CGvHD im jeweils betroffenen Organ. Für das Einzelorgan wird eine Skala von 0-3 verwendet, wobei 0 eine fehlende und 3 eine ausgeprägte Manifestation in dem entsprechenden Organ bedeutet. An der Haut ergibt sich die Schweregradeinteilung sowohl durch die Art der vorhandenen Merkmale als auch durch deren Ausdehnung. So ist bei Vorhandensein einer Sklerose mindestens ein Score von 2 anzunehmen. Ein Hautbefall von $>50 \%$ der Körperoberfläche ist unabhängig davon, ob eine sklerodermiforme oder lichenoide Manifestation vorliegt, als Score 3 zu bewerten [20] ( $\odot$ Tab. 6).

Der Gesamtschweregrad der CGvHD hat 3 Stufen: mild, moderat und schwer. Eine milde cGvHD liegt vor, wenn maximal 2 Organe mit einem maximalen Score von 1 in den entsprechenden Organen betroffen sind. Eine Ausnahme stellt die Lunge dar. Die Diagnose einer pulmonalen cGvHD mit einem Score von 1 führt zur Diagnose einer moderaten cGvHD. Wenn in mindestens 1 Organ ein Score von 2 erhoben wurde oder ein Multiorganbefall vorliegt (3 oder mehr Organe mit einem Score von 1 betroffen), liegt eine moderate cGvHD vor. Eine schwere cGvHD wird diagnostiziert, wenn in mindestens 1 Organ ein Score von 3 oder in der Lunge ein Score von $\geq 2$ vorliegt [20].

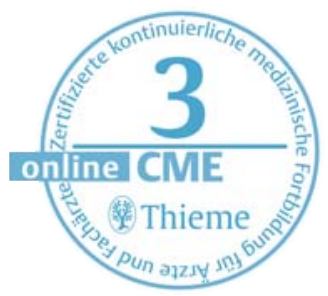

\section{Histologie}

$\checkmark$

Die histopathologischen Merkmale und Kriterien für eine aGvHD wurden bereits 1974 beschrieben [29]. Die aGvHD ist durch eine vakuoläre Interfacedermatitis gekennzeichnet ( $\bullet$ Abb.5). Je nach Schweregrad findet man nur eine Vakuolisation der Basalzellschicht verbunden mit einer spärlichen oberflächlichen lymphozytären Entzündung (Grad 1), Keratinozytenapoptosen ggf. mit anlagernden Lymphozyten (sogenannte Satellitennekrosen) (Grad 2), parzielle Nekrose der Epidermis mit beginnender Kontinuitätstrennung zwischen Epidermis und Korium (Grad 3) und Abhebung der nekrotischen Epidermis (Grad 4), ein Bild, das histologisch dem der toxischen epidermalen Nekrolyse entspricht. Typisch ist die Beteiligung der Schweißdrüsenstrukturen und des Haarfollikelepithels. Die cGvHD kann sich ebenfalls unter dem Bild einer vakuolären Interfacedermatitis manifestieren, sodass eine Abgrenzung zur aGvHD nicht sicher möglich ist. Die NIH-Arbeitsgruppe hat Minimalkriterien, die zur Diagnosestellung einer GvHD erforderlich sind, definiert [30]. Diese umfassen Apoptosen in der Basalzellschicht oder dem Stratum spinosum, dem Haarfollikel oder Akrosyringium \pm ein lichenoides Entzündungsinfiltrat \pm vakuoläre Veränderungen \pm Satellitennekrosen. Die Diagnose einer cGvHD kann anhand weiterer Merkmale spezifiziert werden [30].

\section{Therapie der GuHD}

\section{aGvHD}

Nach der Transplantation wird eine Prophylaxe zur Prävention der aGvHD eingeleitet. Das am meisten eingesetzte Schema besteht aus einem Calcineurininhibitor (Cyclosporin A [CSA] oder Tacrolimus) und Methotrexat. Andere Medikamente, die zur GvHD-Prophylaxe eingesetzt werden, sind u.a. Sirolimus, Mycophenolatmofetil (MMF), Antithymozytenglobulin (ATG) [31,32].

\section{Erstlinientherapie}

Die erfolgreiche Therapie der aGvHD ist von zentraler Bedeutung, da das Ansprechen auf die Erstlinientherapie wesentlich im Hinblick auf das Langzeitüberleben ist. Die Standardtherapie umfasst hoch dosierte Kortikosteroide (CS) $(2 \mathrm{mg} / \mathrm{kg}$ KG Methylprednisolon/die), kombiniert mit einem Calcineurininhibitor (CNI) für 7-14 Tage $[31,32]$. Es konnte gezeigt werden, dass eine höhere Dosis ( $10 \mathrm{mg} / \mathrm{kg}$ KG Methylprednisolon/die) keinen Vorteil gegenüber dieser Dosierung brachte. Eine komplette Remission wird in $25-40 \%$ der Patienten mit einer aGvHD Grad II-IV erreicht [31]. Mit zunehmendem Schweregrad nimmt die Ansprechrate ab. Eine Zweitlinientherapie wird eingeleitet, wenn nach 3 Tagen ein Progress eintritt, nach 5-7 Tagen kein Ansprechen erkennbar 


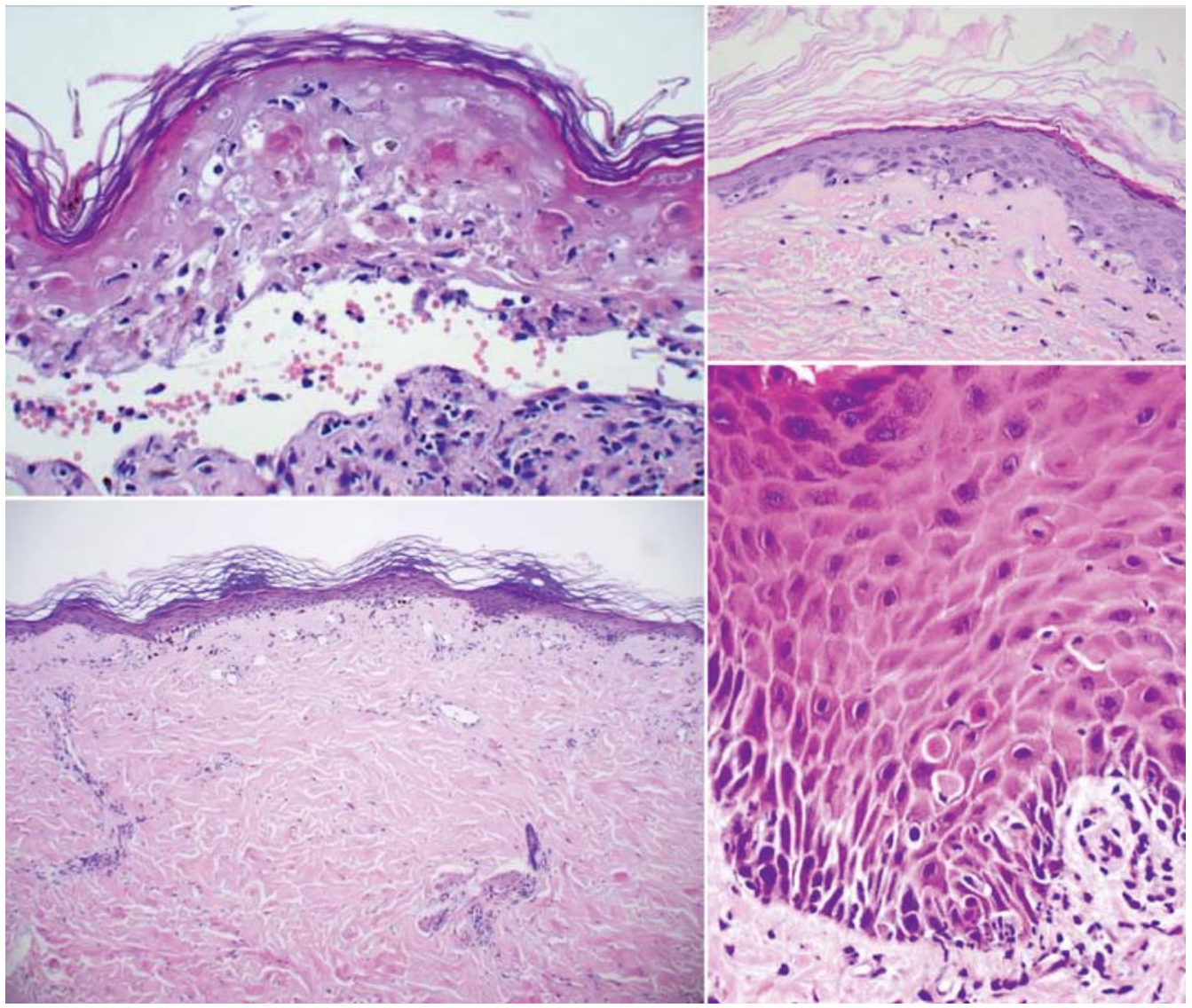

ist und wenn nach 14 Tagen keine komplette Remission eingetreten ist $[31,32]$.

\section{Zweitlinientherapie}

In der Zweitlinientherapie werden Immunsuppressiva wie Sirolimus, MMF oder Tacrolimus, sofern sie in der Prophylaxe nicht bereits appliziert wurden, ATG, Pentostatin sowie verschiedene Antikörper (AK) (Anti-IL-2-Rezeptor-[CD25]-AK, Anti-TNF- $\alpha-A K$, Anti-CD52-AK, Anti-CD147-AK, Anti-CD3-AK) und mesenchymale Stammzellen eingesetzt [31-33].

Bei aGvHD der Haut stellt die Lichttherapie eine Option dar. In einer Studie wurden 103 Patienten mit vorwiegend kutaner steroidresistenter aGvHD mit PUVA behandelt [34]. Die Therapie erfolgte nach Protokoll über 6 Wochen. 65 der 103 Patienten wurden über 6 Wochen behandelt, davon war bei 41 (63\%) am Ende der Therapiephase die Haut-GvHD komplett rückgebildet oder es lag maximal ein Stadium 1 vor. Die Prednisondosis konnte im Mittel von 1,6 auf 0,7 mg/kg KG reduziert werden. In einer Untersuchung an 70 Patienten mit aGvHD der Haut, die eine UVA-1-Therapie erhielten, zeigten $70 \%$ eine Vollremission und $24,3 \%$ eine parzielle Remission [35]. In der bisher größten Untersuchung zur Wirksamkeit der Fotopherese (ECP) wurden prospektiv 59 Patienten mit einer steroidrefraktären oder -abhängigen aGvHD behandelt [36]. Eine komplette Remission konnte bei $82 \%$ der Patienten mit kutaner Mani- festation, bei $61 \%$ mit hepatischer und enteraler Manifestation erreicht werden. Die CS-Therapie konnte im Median nach 55 Tagen (17-284 Tage) beendet werden [36,37]. Patienten, die eine komplette Remission zeigten, hatten eine statistisch signifikant verlängerte Überlebenszeit im Vergleich zu den Patienten, die keine komplette Remission erreichten. Das maximale Ansprechen war im Median bereits nach 1,3 Monaten (0,5-6 Monaten) zu beobachten.

Die Basis der pharmakologischen Therapie der aGvHD sind hoch dosierte Kortikosteroide.

\section{cGvHD}

\section{Erstlinientherapie}

Sobald die Diagnose einer GvHD gesichert ist, muss geprüft werden, ob eine klassische cGvHD vorliegt, ein Overlap-Syndrom oder eine späte aGvHD. Die Therapiestandards für das OverlapSyndrom müssen noch definiert werden. Möglicherweise haben Patienten mit einem OverlapSyndrom eine schlechtere Prognose als Patienten mit klassischer cGvHD, die Datenlage ist aber bisher nicht eindeutig $[38,39]$. Ein Overlap-Syndrom, das sich überwiegend unter dem Bild einer aGvHD manifestiert, wird wie eine aGvHD behandelt [40]. Die Therapie der cGvHD richtet sich nach dem Schweregrad. Nach NIH-Empfehlungen sollte eine systemische immunsuppressive Thera-
Abb. 5 Histologie der GvHD: akute GvHD Grad 4 mit Kontinuitätstrennung zwischen Epidermis und Korium und Nekrose der Epidermis (oben links), Overlap-Syndrom mit vakuoliger Alteration der Basalzellschicht und diskreter Fibrose des Papillarkörpers (oben rechts), chronische sklerodermiforme GvHD mit Ummauerung der Adnexstrukturen in der Dermis und noch vorhandener vakuoliger Alteration der Basalzellschicht und Melanophagen (unten links), Keratinozytenapoptosen mit Satellitennekrose bei chronischer GvHD (unten rechts).

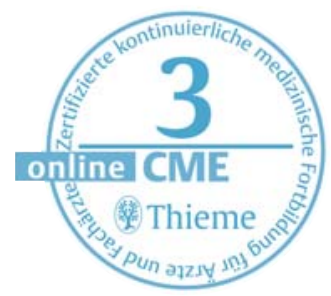


pie (SIT) in Betracht gezogen werden, wenn 3 oder mehr Organe betroffen sind oder in einem Organ ein Score von $\geq 2$ vorhanden ist $[20,26]$. Eine milde cGvHD kann prinzipiell topisch behandelt werden. Zur topischen Therapie der milden cGvHD der Haut werden in der Regel CS oder CNI eingesetzt. Zu CNI liegen bisher nur kleinere Fallserien vor. Elad et al. [41] behandelten 10 Patienten mit einer steroidabhängigen kutanen cGvHD mit Tacrolimus und beobachteten bei 10 Patienten ein Ansprechen auf die Therapie. Die Therapie mit Tacrolimus zeigte in einer anderen Untersuchung ein rasches Ansprechen und kann somit auch unterstützend eingesetzt werden, um eine Symptomlinderung bis zum Greifen weiterer Therapien herbeizuführen [42]. Darüber hinaus kann zur Behandlung der kutanen cGvHD eine Fototherapie (PUVA, UVB, UVA-1-Therapie) eingesetzt werden.

Bei einer milden cGvHD kann eine ausschließliche topische Therapie erfolgreich sein. Zur organgerichteten Therapie der kutanen cGvHD werden topische Kortikosteroide, Calcineurinantagonisten und fototherapeutische Verfahren eingesetzt.

Bei hepatischer Manifestation oder Fasziitis wird auch bei milder cGvHD eine Systemtherapie empfohlen [40]. Liegen bei einer milden cGvHD Faktoren, die mit einer schlechteren Prognose assoziiert sind vor (z. B. Thrombopenie $<100000 / \mu l$ bei Diagnosestellung, progressive onset disease), sollte eine SIT in Betracht gezogen werden [26]. Die SIT wird mit $1 \mathrm{mg} / \mathrm{kg}$ Prednison oder einer Äquivalentdosis von Methylprednisolon begonnen, sofern keine Kontraindikationen infolge von Komorbiditäten vorliegen [26]. Wenn der Patient auf die Therapie mit einer Reduktion der Symptome oder Stabilisierung anspricht, kann nach 2 Wochen mit der schrittweisen Dosisreduktion begonnen werden. Ein mögliches Schema ist die Reduktion der Prednisondosis um 25\% pro Woche mit dem Ziel, eine Dosis $1 \mathrm{mg} / \mathrm{kg}$ alle 2 Tage nach 6-8 Wochen zu erreichen. Diese Dosis wird bei Patienten mit einer schweren cGvHD, bei Vorliegen von Risikofaktoren oder bei Nichterreichen einer Vollremission über 2-3 Monate gehalten und erst dann weiter ausgeschlichen. In einer Kohorte von 330 Patienten, die zwischen 1994 und 2000 in Seattle transplantiert wurden, sprach ein Drittel auf die Initialtherapie an [26]. Der Stellenwert der CS in der Erstlinientherapie wird durch frühere Untersuchungen unterstrichen. Koc et al. [43] führten ein Studie durch, in der bei Patienten mit cGvHD (extensive cGvHD, Thrombozyten $>100000 / \mu l$ ) entweder mit Prednison $1 \mathrm{mg} / \mathrm{kg}$ plus Placebo oder zusätzlich mit CSA $6 \mathrm{mg} / \mathrm{kg}$ alle 2 Tage behandelt wurden. Die Kombinationstherapie führte zwar zur Reduktion von steroidbedingten Komplikationen (aseptische Knochennekrosen), jedoch wurde die transplantationsassoziierte Mortalität nicht reduziert.
Die Kombinationstherapie bei Patienten mit Standardrisiko wird daher nur im Falle zu erwartender Komplikationen der Steroidtherapie empfohlen [40]. Bei Patienten mit schwerer cGvHD, die eine SIT über einen längeren Zeitraum benötigen, kann die Kombinationstherapie mit CSA von Vorteil sein, um Steroide einzusparen und deren Toxizität zu reduzieren [40]. Weitere Medikamente, die in Kombination mit CS eingesetzt werden, sind MMF und mTOR-Inhibitoren. Aufgrund ihrer guten Verträglichkeit stellt die ECP, die bisher lediglich als Zweitlinientherapie bei cGvHD eingesetzt wurde, möglicherweise eine gute Ergänzung zu CS dar, zumal die ECP offensichtlich den GVL-Effekt nicht negativ beeinflusst und nicht mit einem erhöhten Infektionsrisiko einhergeht.

Die pharmakologische Systemtherapie der CGvHD ist kortikosteroidbasiert.

\section{Zweitlinientherapie}

Eine Zweitlinientherapie ist erforderlich, wenn die Patienten auf die initiale Therapie mit CS nicht ansprechen oder Nebenwirkungen zeigen, die eine Therapieumstellung erfordern. Es existiert keine einheitliche Definition der Steroidabhängigkeit bzw. -resistenz, weitgehend akzeptierte Kriterien dafür sind jedoch [44]:

1. Zunahme der Symptome nach 14-tägiger Prednison-Therapie mit $1 \mathrm{mg} / \mathrm{kg}$ KG/die.

2. Keine Besserung (stable Disease) nach 4-8-wöchiger Therapie von mindestens $0,5 \mathrm{mg} / \mathrm{kg} \mathrm{KG} / \mathrm{die}$.

3. Die Reduktion der Prednisondosis auf weniger als $0,5 \mathrm{mg} / \mathrm{kg} \mathrm{KG} /$ die ist nicht möglich (Steroidabhängigkeit).

Es wird empfohlen, bei Einleitung der Zweitlinientherapie auf Medikamente/Verfahren zurückzugreifen, die eine nachgewiesen Effektivität bei akzeptablem Nebenwirkungsprofil haben. Dazu gehören CNI, MMF, ECP und mTOR-Inhibitoren [44]. Zeigt sich nach 4 Wochen ein Progress, wird eine erneute Therapiemodifikation erforderlich, ansonsten wird der Erfolg der Salvagetherapie wie bei der Erstlinientherapie nach 8-12 Wochen beurteilt. $\mathrm{Zu}$ berücksichtigen ist, dass unterschiedliche Organe bzw. unterschiedliche Symptome eine unterschiedliche Ansprechzeit haben. Bei sklerodermiformer kutaner cGvHD kann sich der Therapieerfolg u.U. erst nach 6 Monaten einstellen.

Aufgrund ihrer guten Verträglichkeit wird empfohlen, die ECP als erste Wahl für die Zweitlinientherapie bei steroidrefraktären Patienten zu prüfen. Es liegen zahlreiche Untersuchungen vor, die einen positiven Effekt der ECP auf die cGvHD zeigen. Meist handelt es sich um retrospektive Studien. In der größten retrospektiven Untersuchung analysierten Couriel et al. [45] 71 Patienten mit steroidrefraktärer cGvHD. Es zeigte sich ein organabhängig unterschiedliches Ansprechen auf 
die Therapie. Die Ansprechrate bei kutaner Manifestation betrug 59\%. Von den 21 Patienten mit einer sklerodermiformen kutanen GvHD sprachen $67 \%$ an. Die Ansprechrate bei Mundschleimhautbeteiligung betrug $77 \%$. In der bisher einzigen prospektiven randomisierten Phase-II-Studie wurde bei 95 Patienten mit einer steroidabhängigen oder steroidrefraktären cGvHD die Wirkung der SIT mit der SIT plus ECP verglichen [46]. Die ECP wurde zunächst über einen Zeitraum von 12 Wochen durchgeführt. Patienten, die auf die ECP ansprachen, konnten die Therapie um weitere 12 Wochen fortsetzen. Primärer Endpunkt war der Einfluss der ECP auf die kutane Manifestation nach Woche 12. Im Median konnte eine Verbesserung des Hautscores um 14,5\% nach 12 Wochen beobachtet werden, im Kontrollarm betrug diese $8,5 \%$. Der Unterschied verfehlte knapp das Signifikanzniveau. Dagegen konnte ein statistisch signifikanter Unterschied zwischen Therapie- und Kontrollarm nachgewiesen werden, wenn der Hautscore und der steroidsparende Effekt gemeinsam betrachtet wurden. Nach Woche 12 waren im Therapiearm deutlich mehr Patienten, welche eine Einsparung der Steroide um mindestens $50 \%$ und eine Reduktion des Hautscores um mindestens $25 \%$ zeigten. Patienten, die bis zur Woche 24 behandelt wurden, zeigten eine Reduktion des Hautscores um 31,4\%. Weitere Medikamente, die in der Zweitlinientherapie eingesetzt werden, sind Rituximab, Methotrexat, Pentostatin, Alemtuzumab, Etanercept, Alefacept, Clofazimin, Hydroxychloroquin, Thalidomid, Retinoide, Azathioprin und Imatinib $[26,44]$.

Eine wichtige Zweitlinientherapie der cGvHD ist die ECP.

\section{Infektionsprophylaxe}

Patienten mit einer GvHD sind in erhöhtem Maß gefährdet, eine virale, mykotische oder bakterielle Infektion zu erleiden. Aufgrund der Immunsuppression besteht die Gefahr, dass Infektionen schwerer und potenziell lebensbedrohlich verlaufen. Daher wird ergänzend zu der GvHD-gerichteten Therapie häufig eine Infektionsprophylaxe, z.B. gegen Pneumocystis jiroveci, erforderlich.

\section{Zusammenfassung}

$\nabla$

Die Anzahl der hämatopoetischen Stammzelltransplantationen ist in den vergangenen Jahren stetig gewachsen. Die wesentlichen Indikationen sind Leukämien und maligne Lymphome. Ein wichtiger Therapieeffekt der Stammzelltransplantation ist der sogenannte Graft-versus-Leukemia-Effekt, der darauf beruht, dass immunkompetente Zellen des Spenders Gewebeantigene der Tumorzellen des Empfängers erkennen und sie eliminieren. Eine damit einhergehenden Folge ist die Entwicklung einer GvHD. Die aGvHD, die sich hauptsächlich an Haut, Darm und Leber manifestiert, ist ein potenziell lebensbedrohliches Krankheitsbild, das einer umgehenden Therapie bedarf. Die cGvHD tritt bei 30-70\% der Patienten auf und stellt die schwerwiegendste Langzeitkomplikation der SZT dar. Bisher wurden aGvHD und CGvHD nach ihrem zeitlichen Auftreten nach der Transplantation (aGvHD vor Tag 100 und cGvHD nach Tag 100) unterschieden. Die Einteilung hat kürzlich durch eine NIH-Konsensusempfehlung eine grundlegende Änderung erfahren. Die Unterscheidung in aGvHD und cGvHD richtet sich neben dem Zeitpunkt des Auftretens wesentlich nach den aufgetretenen Symptomen. Anhand der Art und Stärke/Ausdehnung der klinischen Merkmale wird ein Organscore und aus diesem ein Gesamtschweregrad der cGvHD erstellt. Auf der Basis der NIH-Empfehlungen wird in Zukunft eine saubere Klassifizierung der GvHD möglich und damit die Grundlage vorhanden sein, an gut definierten Kollektiven Studien u.a. zur Therapie der GvHD durchzuführen.

\section{Literatur}

1 Hertenstein B, Ganser A. Knochenmarktransplantation. Internist 2004; 45: $1261-1267$

2 Deutsches Register für Stammzelltransplantation. Jahresbericht 2009. Im Internet: www.drst.de/jb.html Stand 11.08.2010

3 Buchholz S, Ganser A. Hämatopoetische Stammzelltransplantation. Internist 2009; 50: 572 - 580

4 Billingham RE. The biology of graft-versus-host reactions. Harvey Lect 1966-1967; 62: 21 - 78

5 Ringdén O, Karlsson H, Olsson R et al. The allogeneic graftversus-cancer effect. Br J Haematol 2009; 147: 614 - 633

6 Ferrara JLM, Levine JE, Reddy $P$ et al. Graft-versus-host disease. Lancet 2009; 373: 1550-1561

7 Shlomchik WD. Graft-versus-host disease. Nat Rev Immunol 2007; 7: 340-352

8 Beelen DW, Haralambie E, Brandt $H$ et al. Evidence that sustained growth suppression of intestinal anaerobic bacteria reduces the risk of acute graft-versus-host disease after sibling marrow transplantation. Blood 1992; 80: $2668-2676$

9 Penack O, Holler E, van den Brink MR. Graft-versus-host disease: regulation by microbe-associated molecules and innate immune receptors. Blood 2010; 115: 1865 1872

10 Leung AY, Kwong YL. Haematopoetic stem cell transplantation: current concepts and novel therapeutic strategies. Br Med Bull 2010; 93: 85-103

11 Ball LM, Egeler RM; EBMT Paediatric Working Party. Acute GvHD: pathogenesis and classification. Bone Marrow Transplant 2008; 41 (Suppl. 2): 58-64

12 Martin PJ. Biology of chronic graft-versus-host disease: implications for a future therapeutic approach. Keio J Med 2008; 57: $177-183$

13 Lee JW, Deeg JH. Prevention of chronic GVHD. Best Pract Res Clin Haematol 2008; 21: 259-270

14 Shimabukuro-Vornhagen A, Hallek MJ, Storb RF et al. The role of $B$ cells in the pathogenesis of graft-versus-host disease. Blood 2009; 114: 4919-4927

15 Svegliati S, Olivieri A, Campelli $N$ et al. Stimulatory autoantibodies to PDGF receptor in patients with extensive chronic graft-versus-host disease. Blood 2007; 110: 237 $-241$

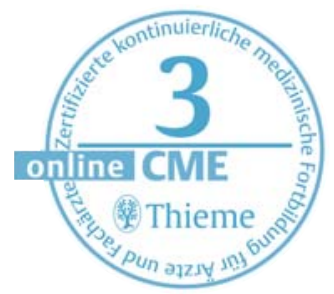


16 Kim SJ, Lee JW, Jung CW et al. Weekly rituximab followed by monthly rituximab treatment for steroid-refractory chronic graft-versus-host disease: results from a prospective, multicenter, phase II study. Haematologica 2010; 95: 1935-1942

17 Rieger K, Loddenkemper C, Maul j et al. Mucosal FOXP3+ regulatory $\mathrm{T}$ cells are numerically deficient in acute and chronic GvHD. Blood 2006; 107: 1717-1723

18 Hoffmann P, Ermann J, Edinger M et al. Donor-type CD4(+) CD25(+) regulatory $T$ cells suppress lethal acute graftversus-host disease after allogeneic bone marrow transplantation. J Exp Med 2002; 196: 389-399

19 Pidala J, Anasetti C. Can antigen-specific regulatory T cells protect against graft versus host disease and spare antimalignancy alloresponse? Haematologica 2010; 95: 660 $-665$

20 Filipovich AH, Weisdorf D, Pavletic S et al. National Institutes of Health consensus development project on criteria for clinical trials in chronic graft-versus-host disease: I. Diagnosis and staging working group report. Biol Blood Marrow Transplant 2005; 11: 945-956

21 Glucksberg H, Storb R, Fefer A et al. Clinical manifestations of graft-versus-host disease in human recipients of marrow from HL-A-matched sibling donors. Transplantation 1974; 18: 295-304

22 Przepiorka D, Weisdorf D, Martin P et al. 1994 Consensus Conference on Acute GVHD Grading. Bone Marrow Transplant 1995; 15: 825-828

23 Cahn JY, Klein JP, Lee SJ et al. Prospective evaluation of 2 acute graft-versus-host (GVHD) grading systems: a joint Société Française de Greffe de Moëlle et Thérapie Cellulaire (SFGM-TC), Dana Farber Cancer Institute (DFCl), and International Bone Marrow Transplant Registry (IBMTR) prospective study. Blood 2005; 106: $1495-$ 1500

24 Socié G, Stone JV, Wingard JR et al. Long-term survival and late deaths after allogeneic bone marrow transplantation. Late Effects Working Committee of the International Bone Marrow Transplant Registry. N Engl J Med 1999; 341: $14-21$

25 Joseph RW, Couriel DR, Komanduri KV. Chronic Graft-Versus-Host Disease After Allogeneic Stem Cell Transplantation: Challenges in Prevention, Science, and Supportive Care. J Support Oncol 2008; 6: 361-372

26 Lee SJ, Flowers MED. Recognizing and Managing Chronic Graft-Versus-Host Disease. Hematology Am Soc Hematol Educ Program 2008; 1: 134-141

27 Baker KS, Fraser Cl. Quality of life and recovery after graftversus-host disease. Best Pract Res Clin Haematol 2008; 21: $333-341$

28 Hymes SR, Turner ML, Champlin RE et al. Cutaneous Manifestations of Chronic Graft-versus-Host Disease. Biol Blood Marrow Transplant 2006; 12: 1101-1113

29 Lerner KG, Kao GF, Storb R et al. Histopathology of graftversus-host reaction (GVHR) in human recipients of marrow from HL-A matched sibling donors. Transplant Proc 1974; 6: 367-371

30 Shulman HM, Kleiner D, Lee S/ et al. Histopathologic diagnosis of chronic graft-versus-host disease: National Institutes of Health Consensus Development Project on Criteria for Clinical Trials in Chronic Graft-versus-Host Disease: II. Pathology Working Group Report. Biol Blood Marrow Transplant 2006; 12: 31-47
31 Apperley J, Carreras E, Gluckman E et al., eds. The EBMTESH Handbook on Haematopoietic stem cell transplantation. 5th ed.; 2008. Im Internet: www.ebmt.org/ EBMT_Handbook.html

32 Deeg HJ. How I treat refractory acute GVHD. Blood 2007; 109: 4119-4126

33 Paczesny S, Choi SW, Ferrara JL. Acute graft-versus-host disease: new treatment strategies. Curr Opin Hematol 2009; 16: 427-436

34 Furlong T, Leisenring W, Storb R et al. Psoralen and Ultraviolet A Irradiation (PUVA) as Therapy for Steroid-Resistant Cutaneous Acute Graft-versus-Host Disease. Biol Blood Marrow Transplant 2002; 8: 206-212

35 Schlaak M, Schwind S, Wetzig T et al. UVA (UVA-1) therapy for the treatment of acute GVHD of the skin. Bone Marrow Transplant 2010; 45: 1741 - 1748

36 Greinix HT, Knobler RM, Worel N et al. The effect of intensified extracorporeal photochemotherapy on long-term survival in patients with severe acute graft-versus-host disease. Haematologica 2006; 91: 405-408

37 Greinix HT, Worel N, Knobler R. Role of Extracorporeal Photopheresis (ECP) in treatment of steroidrefractory acute graft-versus-host disease. Biol Blood Marrow Transplant 2010; 16: $1747-1748$

38 Jagasia M, Giglia J, Chinratanalab $W$ et al. Incidence and outcome of chronic graft-versus-host disease using National Institutes of Health consensus criteria. Biol Blood Marrow Transplant 2007; 13: 1207- 1215

39 Cho BS, Min CK, Eom KS et al. Feasibility of NIH consensus criteria for chronic graft-versus-host disease. Leukemia 2009; 23: $78-84$

40 Wolff $D$, Gerbitz A, Ayuk F et al. Consensus conference on clinical practice in chronic graft-versus-host disease (GVHD): First-line and topical treatment of chronic GVHD. Biol Blood Marrow Transplant 2010; 16: 1611 1628

41 Elad S, Or R, Resnick I, Shapira MY. Topical tacrolimus - a novel treatment alternative for cutaneous chronic graftversus-host disease. Transpl Int 2003; 16: 665-670

42 Choi Cl, Nghiem P. Tacrolimus ointment in the treatment of chronic cutaneous graft-vs-host disease: a case series of 18 patients. Arch Dermatol 2001; 137: $1202-1206$

43 Koc S, Leisenring W, Flowers ME et al. Therapy for chronic graft-versus-host disease: a randomized trial comparing cyclosporine plus prednisone versus prednisone alone. Blood 2002; 100: 48 - 51

44 Wolff $D$, Schleuning $M$, von Harsdorf $S$ et al. Consensus conference on clinical practice in chronic GVHD: Second-Line Treatment of chronic Graft-versus-Host Disease. Biol Blood Marrow Transplant 2011; 17: 1-17

45 Couriel DR, Hosing C, Saliba R et al. Extracorporeal photochemotherapy for the treatment of steroid-resistant chronic GVHD. Blood 2006; 107: 3074- 3080

46 Flowers ME, Apperley JF, van Besien K et al. A multicenter prospective phase 2 randomized study of extracorporeal photopheresis for treatment of chronic graft-versus-host disease. Blood 2008; 112: 2667-2674

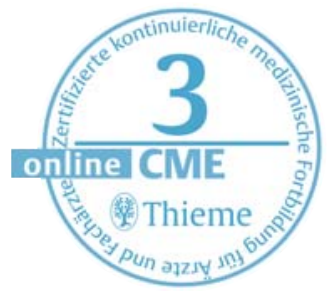




\section{CME-Fragen Graft-versus-Host-Erkrankung der Haut}

1 Welche Aussage trifft zu? Eine Graft-versus-Host-Erkrankung kann sich entwickeln:

A nur, wenn der Spender mit dem Empfänger verwandt ist.

B nur, wenn der Spender nicht mit dem Empfänger verwandt ist.

C nur, wenn Spender und Empfänger nicht HLA-identisch sind.

D auch bei HLA-identischer Transplantation.

E im Zuge der Abstoßung des Transplantats durch den Empfänger.

2 Welche Aussage ist falsch? Die späte akute Graft-versusHost-Erkrankung

A tritt später als 100 Tage nach der Transplantation auf.

B definiert sich im Gegensatz zum Overlap-Syndrom auch über den zeitlichen Abstand zur Transplantation.

C definiert sich über den Zeitpunkt ihres Auftretens nach Transplantation und den klinischen Symptomen.

D weist im Gegensatz zum Overlap-Syndrom keine Zeichen der chronischen Graft-versus-Host-Erkrankung auf.

E unterscheidet sich von der klassischen akuten Graft-versus-Host-Erkrankung durch ihre klinischen Zeichen.

3 Welche Aussage trifft zu? Was gehört zu den diagnosti-

schen Zeichen der chronischen Graft-versus-Host-Erkrankung?

A Longitudinalriffelung der Nägel

B Mukositis

C neu aufgetretene vernarbende Alopezie

D neu aufgetretene nicht vernarbende Alopezie

E Poikilodermie

4 Bei welcher der nachfolgenden Konstellationen kann die Diagnose einer chronischen Graft-versus-Host-Erkrankung gemäß NIH-Konsensus gestellt werden?

A Neu aufgetretene nicht vernarbende Alopezie mit histologischem Nachweis einer oberflächlichen perivaskulären Dermatitis

B Makulopapulöses Exanthem und Onycholyse

C Makulopapulöses Exanthem und vernarbende Konjunktivitis

D Netzartige Zeichnung der Wangenschleimhaut mit Nachweis einer Interfacedermatitis mit Keratinozytenapoptosen

E Keratosis-pilaris-artige Veränderungen und Onycholyse
5 Welches der genannten Symptome gilt als charakteristisch für eine klassische akute Graft-versus-Host-Erkrankung? ein palmoplantares Erythem

B ein an der Kopfhaut beginnendes morbilliformes Exanthem

C lichenoide Papeln an den Handrücken

D eine narbig abheilende Ösophagitis

E eine Bronchiolitis obliterans

6 Was kann im Rahmen der klassischen chronischen Graft-versus-Host-Erkrankung nicht vorkommen? perifollikuläre Erytheme Xerosis cutis, teils ichthyosiform makulopapulöse Exantheme

D Dermatomyositis-artige livide Erytheme an den Händen E ein der toxischen epidermalen Nekrolyse ähnliches Krankheitsbild

7 Eine kutane chronische Graft-versus-Host-Erkrankung mit Score 3 liegt vor bei

A lichenoiden Papeln mit Beteiligung auf 30\% der Körperoberfläche

B lichenoiden Papeln mit Beteiligung auf $51 \%$ der Körperoberfläche

C oberflächlicher Sklerose auf 20\% der Körperoberfläche

D oberflächlicher Sklerose auf 15\% der Körperoberfläche

E oberflächlicher Sklerose auf 10\% der Körperoberfläche

8 Welche Aussage trifft zu? Ein Stadium 3 der akuten Graft-versus-Host-Erkrankung liegt vor bei einem makulopapulösen Exanthem $<25 \% \mathrm{KOF}$ einem makulopapulösen Exanthem 25\%-50\% KOF einem makulopapulösen Exanthem > 50\% KOF/Erythrodermie

D Erythrodermie mit Blasen/Epidermolyse

E intermittierende Übelkeit 
9 Welche Aussage trifft zu? Die PUVA-Therapie bei akuter Graft-versus-Host-Erkrankung ist nicht indiziert wegen der zu erwartenden unerwünschten Wirkungen.

B ist dann indiziert, wenn die Standardtherapie mit $10 \mathrm{mg} / \mathrm{kg}$ KG Methylprednisolon/die nicht wirksam ist.

C wurde bereits eingesetzt und zeigte einen steroidsparenden Effekt.

D wurde bereits eingesetzt, zeigte aber keinen steroidsparenden Effekt.

E wird im Rahmen der Prophylaxe nicht zur Therapie eingesetzt.
10

A

B

C

$E$
Welche Aussage trifft zu? Bei der kutanen chronischen Graft-versus-Host-Erkrankung

ist eine ausschließliche topische Therapie gerechtfertigt. muss systemisch mit $0,5 \mathrm{mg} / \mathrm{kg}$ KG Prednison therapiert werden.

muss systemisch mit $1 \mathrm{mg} / \mathrm{kg}$ KG Prednison therapiert werden.

muss systemisch mit Prednison in Kombination mit Cyclosporin therapiert werden.

muss systemisch mit Prednison in Kombination mit einer Lichttherapie behandelt werden.

\section{CME-Fortbildung mit der Aktuellen Dermatologie}

Zertifizierte Fortbildung Hinter der Abkürzung CME verbirgt sich „continuing medical education“, also kontinuierliche medizinische Fort- und Weiterbildung. Zur Dokumentation der kontinuierlichen Fortbildung der Ärzte wurde das Fortbildungszertifikat der Ärztekammern etabliert. Hauptzielgruppe für das Fortbildungszertifikat sind Ärzte mit abgeschlossener Facharztausbildung, die im 5-jährigen Turnus einen Fortbildungsnachweis erbringen müssen. Es ist jedoch auch für Ärzte im Praktikum bzw. in der Facharztweiterbildung gedacht.

Die Fortbildungseinheit In den einheitlichen Bewertungskriterien der Bundesärztekammer ist festgelegt: „Die Grundeinheit der Fortbildungsaktivitäten ist der Fortbildungspunkt. Dieser entspricht in der Regel einer abgeschlossenen Fortbildungsstunde (45 Minuten)“. Für die erworbenen Fortbildungspunkte muss ein Nachweis erbracht werden. Hat man die erforderliche Anzahl von 250 Punkten gesammelt, kann man das Fortbildungszertifikat bei seiner Ärztekammer beantragen, welches man wiederum bei der KV (niedergelassene Ärzte) oder bei seinem Klinikträger (Klinikärzte) vorlegen muss.

Anerkennung der CME-Beiträge Die Fortbildung in der Aktuellen Dermatologie wurde von der Nordrheinischen Akademie für ärztliche Fort- und Weiterbildung für das Fortbildungszertifikat anerkannt, das heißt, die Vergabe der Punkte kann direkt durch die Thieme Verlagsgruppe erfolgen. Die Fortbildung in der Aktuellen Dermatologie gehört zur Kategorie „strukturierte interaktive Fortbildung“. Entsprechend einer Absprache der Ärztekammern werden die von der Nordrheinischen Akademie für ärztliche Fort- und Weiterbildung anerkannten Fortbildungsveranstaltungen auch von den anderen zertifizierenden Ärztekammern anerkannt.
Datenschutz Ihre Daten werden ausschließlich für die Bearbeitung dieser Fortbildungseinheit verwendet. Es erfolgt keine Speicherung der Ergebnisse über die für die Bearbeitung der Fortbildungseinheit notwendige Zeit hinaus. Die Daten werden nach Versand der Testate anonymisiert. Namens- und Adressangaben dienen nur dem Versand der Testate. Die Angaben zur Person dienen nur statistischen Zwecken und werden von den Adressangaben getrennt und anonymisiert verarbeitet.

Teilnahme Jede Ärztin und jeder Arzt soll das Fortbildungszertifikat erlangen können. Deshalb ist die Teilnahme am CME-Programm der Aktuellen Dermatologie nicht an ein Abonnement geknüpft! Die Teilnahme ist im Internet unter http://cme.thieme. de möglich. Man muss sich registrieren, wobei die Teilnahme an Fortbildungen abonnierter Zeitschriften ohne Zusatzkosten möglich ist.

Teilnahmebedingungen Für eine Fortbildungseinheit erhalten Sie 3 Fortbildungspunkte im Rahmen des Fortbildungszertifikates. Hierfür müssen $70 \%$ der Fragen richtig beantwortet sein.

CME-Fortbildung für Nicht-Abonnenten Teilnehmer, die nicht Abonnenten der Aktuellen Dermatologie sind, können für die Internet-Teilnahme dort direkt ein Guthaben einrichten, von dem pro Teilnahme ein Unkostenbeitrag abgebucht wird.

\section{Teilnahme online unter http://cme.thieme.de}

